\title{
Aplicación del criterio D-Óptimo a la fabricación del bloque Samo \#4 para proponer una mezcla bajo estándares NTC4205-1
}

\author{
D-Optimal Criterion Application to Block Samo \# 4 Manufacturing to Propose a \\ Mixing Under Standards NTC4205-1
}

\author{
K. F. Gil-Rodríguez iD ; J. A. Mendoza-Ruíz iD , A. Saltarín-Jiménez iD ; M. Coba-Salcedo iD; \\ J. Roldán-Mckinley iD
}

\begin{abstract}
Resumen-Se aplica el diseño de experimentos para encontrar la proporción óptima de componentes para mezcla del bloque samo \#4 en la empresa Gres Caribe S.A. (Barranquilla, Colombia). Se consideraron los estándares NTC 4205-1 para resistencia a la compresión y absorción de agua. El análisis estadístico de los datos recolectados fue asistido con Statgraphics Centurion XVI. Las pruebas de campo preliminares establecieron los límites de cada componente para determinar la región experimental. Los puntos candidatos se seleccionaron bajo el criterio D-optimo y el modelo de regresión escogido. En total resultan veinte puntos, o mezclas candidatas, que indican que se requirieron veinte corridas para el experimento. Se determinó la mezcla óptima para el bloque, a través de la maximización de la función de deseabilidad, con $54.3091 \%$ arcilla, $37.5507 \%$ arena y $\mathbf{8 . 1 4 0 1 7 \%}$ agua. Cinco corridas de campo con esta mezcla mostraron que los valores de propiedades de los bloques excedían las propiedades de los bloques fabricados con la antigua mezcla, además de cumplir con los estándares requeridos. Se logra un ahorro de $\$ 9.606 /$ bloque fabricado, equivalente a una disminución porcentual de $4.41 \%$, cuantificada en \$153'023.580/año.
\end{abstract}

Palabras claves - Absorción de agua; Arcilla; D-óptimo; Proporciones; Resistencia a la compresión; Superficie de respuesta.

Este manuscrito fue enviado el 05 de agosto de 2019 y aceptado el 27 de marzo de 2020.

Este trabajo fue patrocinado por la Vicerrectoría de Investigaciones, Extensión y Proyección Social de la Universidad del Atlántico (Puerto Colombia, Colombia.

K. Gil-Rodríguez, Ingeniero Mecánico egresado de la Universidad del Atlántico, Carrera 30 \# 8-49, Puerto Colombia, Colombia (e-mail: ing_kevin_gil@hotmail.com).

J. Mendoza-Ruiz, Ingeniero Mecánico egresado de la Universidad del Atlántico, Carrera 30 \# 8-49, Puerto Colombia, Colombia (e-mail: jaldairmendoza@mail.uniatlantico.edu.co).

A. Saltarín-Jiménez, Profesor Auxiliar en la Universidad del Atlántico, Programa de Ingeniería Mecánica, Carrera 30 \# 8-49, Puerto Colombia, Colombia (e-mail: ing_saltarin@yahoo.com).

M. Coba-Salcedo, Profesor Titular en la Universidad del Atlántico, Programa de Ingeniería Mecánica, Carrera 30 \# 8-49, Puerto Colombia, Colombia (e-mail: miltoncoba@uniatlantico.edu.co).

J. Roldán-Mckinley, Profesor Titular en la Universidad del Atlántico, Programa de Ingeniería Mecánica, Carrera 30 \# 8-49, Puerto Colombia, Colombia (e-mail: javierroldan@uniatlantico.edu.co).
Abstract-The design of experiments is applied to find the optimum proportion of components for the samo \# 4 block mix in the company Gres Caribe S.A. (Barranquilla, Colombia). The NTC 4205-1 standards for resistance to compression and water absorption were considered. Statistical analysis of collected data was assisted with Statgraphics Centurion XVI. Preliminary field tests established the limits of each component to determine the experimental region. The candidate points were selected under the D-optimal criterion and the regression model chosen. There are twenty points (o candidate mixtures) in total, thus twenty runs were required for the experiment. By maximizing the desirability function, the optimal mixture for the block was determined: $\mathbf{5 4 . 3 0 9 1 \%}$ clay, $\mathbf{3 7 . 5 5 0 7 \%}$ sand and $8.14017 \%$ water. Five field runs with this mixture showed that the property values of the new blocks exceeded the properties of the blocks manufactured with the old mixture. These blocks also meet the required standards. A savings of \$ 9,606 / manufactured block is achieved, equivalent to a percentage decrease of $4.41 \%$, quantified at COP $153{ }^{\prime} 023,580$ / year.

Index terms - Water absorption; Clay; D-optimal, Proportions; Compression resistance; Response surface.

\section{INTRODUCCIÓN}

$\mathrm{L}^{\mathrm{a}}$ A experimentación juega un papel fundamental en el campo de la industria, es frecuente que se realicen experimentos siempre que se requiera resolver un problema o comprobar alguna idea, como realizar cambios en los materiales de un producto, modificar las condiciones de operación en un proceso, encontrar la temperatura óptima de trabajo de una máquina, etc. [1]. Por ende, es necesario introducir una estrategia para la experimentación, conocida como Diseño de Experimentos, una metodología estadística que permite planificar y realizar eficientemente los experimentos y luego analizar los datos resultantes a fin de obtener conclusiones válidas y objetivas [2].

La primera vez que se aplicó estadística a la experimentación fue en 1920, cuando Ronald A. Fisher fue contratado en Rothamsted Experimental Station para realizar un minucioso análisis estadístico a los datos recolectados de las investigaciones en plantaciones de trigo. En este período Fisher desarrolló el análisis de varianza y el diseño factorial [3]. En 1958 Scheffé introdujo la teoría de mezclas, una metodología estadística capaz de estudiar el comportamiento 
de una variable de respuesta que depende de la proporción de los componentes de una mezcla, manteniendo constante la cantidad total de la misma [4].Los diseños de experimentos con mezclas, se han utilizado para predecir de manera efectiva, los efectos que los cambios en las materias primas o en sus proporciones pueden ejercer sobre las variables del proceso y como estos afectan al producto final. Estos se han aplicado con éxito en la optimización de formulaciones en la industria alimentaria, de pinturas, de medicamentos, de polímeros, de productos cerámicos, entre otras [5] [6] [7].

En la industria de productos cerámicos, específicamente en los ladrillos, el diseño de experimentos con mezclas ha venido aplicándose para obtener un producto final que cumpla con las especificaciones mínimas exigidas y que, además, reduzca su costo de fabricación. Álvarez obtuvo la proporción óptima de mezclado de arcilla y arena para la fabricación de ladrillos artesanales en la región de Cuenca-Ecuador, utilizó una mezcla de dos componentes para la cual la región simplex está dada por una línea recta [8] [9]. Correa et al. modelaron la resistencia a la flexión y la contracción lineal en la cocción de ladrillos cerámicos, en función del porcentaje en la mezcla de tres arcillas de composición diferente, utilizaron una región simplex-centroid lattice y la metodología de superficies de respuesta para calcular el modelo de regresión relativo a la contracción en la cocción [10]. Otros autores han incursionado en estudio de materiales más amigables con el ambiente para la fabricación de ladrillos, como Onwuka, Chijioke y Onwuka que desarrollaron un modelo basado en una región simplex, para describir el comportamiento de la resistencia a la compresión en ladrillos con adición de ceniza de aserrín (SDA), el cual es un residuo industrial generado por la industria de la madera [11]. Posteriormente, se desarrolló un programa computacional basado en las teorías de región simplex y de la función de respuesta derivada de la teoría de regresión, para determinar la proporción de arcilla, SDA y agua necesaria para obtener un valor de resistencia a la compresión deseado, el lenguaje de programación utilizado fue Visual Basic 6.0 [12].

En muchas ocasiones existen restricciones en las proporciones de los componentes de la mezcla, ya sea por condiciones inherentes del proceso o por la experiencia recolectada a lo largo de los años. Estas restricciones modifican la forma de la región simplex, resultando en la mayoría de los casos un polígono o hiperpolígono irregular, que imposibilita usar los modelos del tipo simplex, por lo que se debe recurrir a otros criterios como el D-óptimo [13]. Xinghua, Yunhui, Li y Zhenyu utilizaron el diseño de experimentos con mezclas basado en el criterio D-óptimo para desarrollar y optimizar las formulaciones de Gliclazida de liberación prolongada mediante la combinación de dos polímeros hidrofilacios: HPMC K 15M y Alginato de sodio como retardante [14].

En este artículo se plantea un análisis a la problemática actual en la línea de producción del bloque samo \#4 en la empresa Gres Caribe S.A., este producto se ha fabricado de manera empírica desde 1994, y actualmente genera un alto porcentaje de bloques de segunda y de desperdicio, en comparación con los generados en la fabricación de otros productos. Dicho análisis, consiste en la implementación del diseño de experimentos de mezclas basado en el criterio Dóptimo, para establecer una relación cuantitativa entre los componentes del bloque y las propiedades de producto terminado exigidas por la NTC 4205-1, en resistencia a la compresión y absorción de agua y que, además, sea rentable económicamente, teniendo en cuenta los porcentajes de desperdicios y de segunda generados en cada caso a considerar.

Uno de los beneficios logrados con los resultados del análisis fue sentar las bases para una futura certificación de la calidad del producto, y con ello incrementar las ventas y posicionamiento en el mercado. Lo anterior es muy significativo para la empresa, puesto que el sector de la construcción viene en crecimiento $\mathrm{y}$, cada vez demanda más productos de alta calidad; en los últimos reportes del boletín de coyuntura económica de Barranquilla, emitidos por la Cámara de Comercio, el sector presentó en el primer trimestre del 2016 un aumento del 11.5\%, siendo Atlántico el quinto departamento en el país con mayor número de metros cuadrados autorizados para construir [15].

En este artículo se propone una mezcla para la fabricación del bloque samo \#4 en la empresa Gres Caribe S.A. que permita un producto terminado que cumpla los estándares de compresión y absorción de agua de la norma NTC4205-1, mediante el diseño de experimentos con mezclas basado en el criterio D-óptimo. El estudio inicia con la recolección de información del efecto de las variables de entrada (arcilla, arena, agua), sobre las variables de salida (resistencia a la comprensión y coeficiente de absorción de agua), mediante pruebas de campo previas en el proceso de producción del bloque. Posteriormente, son seleccionadas las mezclas bajo el criterio D-óptimo con asistencia de un software de Análisis Estadístico. Teniendo en cuenta los resultados de los ensayos realizados, se realiza la modelación estadística para establecer la nueva composición de la mezcla del bloque, esta debe cumplir con las propiedades deseadas del producto final. Finalmente, se interpretan los resultados obtenidos para las proporciones de mezclas en conjunto con el departamento de producción de la empresa ladrillera.

\section{EXPERIMENTOS PILOTOS EN EL PROCESO DE FABRICACIÓN DEL BLOQUE SAMO \#4}

Las innovaciones tecnológicas actuales han hecho que las industrias tengan un nivel competitivo más exigente en el mercado, por lo que han puesto en marcha planes para mejorar la eficiencia en cada etapa de la producción, esto sin alterar su código técnico. Hoy en día las empresas ladrilleras han tenido muchas dificultades, debido a las nuevas técnicas de construcción que han ido desplazando en gran manera el uso de productos arcillosos por otro tipo de materiales, debido a sus bajos costos comerciales y fácil manipulación. Por lo tanto, se hace necesario la mejora continua en sus procesos, para así, poder ofrecer productos de mejor calidad y con un precio que les permita ser competitivos en el mercado [1].

Gres Caribe S.A es una empresa ubicada en el 
corregimiento de Juan Mina, jurisdicción de la ciudad de Barranquilla, la cual se dedica a la fabricación y comercialización de productos de arcilla para la construcción. En la actualidad se presentan problemas en el proceso de fabricación del bloque samo \#4, producto insignia de la empresa. Por lo tanto, atendiendo las condiciones actuales del mercado y la problemática en la línea de producción, se determinó optimizar la mezcla para el bloque, modificando los porcentajes de participación de sus elementos constitutivos. Pero antes de ejecutar el experimento, se deben realizar unas pruebas de campo previas que permitan caracterizar las variables que intervienen en el mismo, y así, poder delimitar las mezclas a una región factible que pueda ser solucionable con eficiencia.

\section{A. Arcilla}

Las arcillas constituyen la principal materia prima para la fabricación de productos cerámicos para la construcción. Esta es una roca sedimentaria descompuesta constituida por agregados de silicatos de aluminio hidratados, procedentes de la descomposición de rocas que contienen feldespato [16]. El feldespato es el mineral más abundante de la corteza terrestre. Sin embargo, es bastante raro encontrar yacimientos de feldespato puro; lo más frecuente es que haya cristalizado simultáneamente con otros minerales formando diferentes rocas: granito, diorita, pegmatita, aplita, andesitas, basaltos, ..., etc. El feldespato, de todas estas rocas puede ser alterado por la intemperie y transformarse en arcilla [17]. El gran agente de esta transformación ha sido el agua. Cuando el agua conteniendo $\mathrm{CO}_{2}$ entra en contacto con la roca feldespática, disuelve y se lleva el $\mathrm{K}_{2} \mathrm{O}$ y parte de la sílice. La sílice y la alúmina restantes, después de un tiempo de contacto con la humedad se hidratan y transforman en arcilla (caolín) [16].

La arcilla estudiada en el presente trabajo proviene de un yacimiento ubicado a $5 \mathrm{~km}$ del corregimiento de cuatro bocas, jurisdicción del municipio de Tubará-Atlántico. La cantera es explotada por Gres Caribe S.A, la cual posee el título minero $\mathrm{N}^{\circ}$ FL3-082 y tiene una licencia ambiental otorgada mediante la resolución $\mathrm{N}^{\circ} 000588$ del 5 de octubre de 2009.

\section{B. Bloque Samo}

El bloque samo es un tipo de ladrillo cerámico usado en construcción. Este tiene forma de paralelepípedo rectangular y es pensado para una fácil manipulación humana. Son fabricados a partir de arcilla, arena, agua u otros aditivos; cocidos a una temperatura suficientemente alta para alcanzar una ligazón cerámica [18]. En la Fig. 1, se observa el aspecto del bloque samo \# 4 .

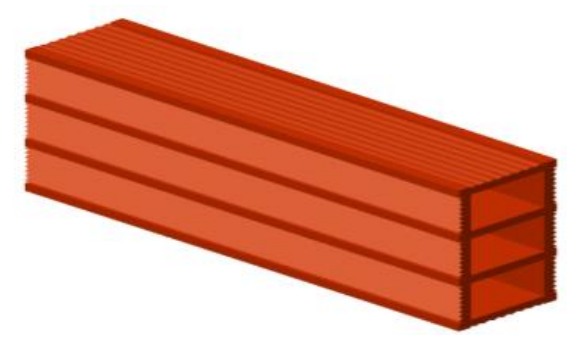

Fig. 1. Aspecto del bloque samo \#4.

\section{Clases de Bloque Samo}

La clasificación de los bloques samo depende del uso en el que se implemente en la construcción; es por esto que dentro de la industria se encuentran diferentes tipos de bloque samo tales como bloque samo \#3, samo \#4, samo \#5 y samo \#6. En la Tabla I, se muestran las dimensiones típicas del bloque tipo samo.

En este proyecto solo se trabajará con el bloque samo \#4, puesto que se genera un alto porcentaje de bloques de segunda y de desperdicio (mayor a 10\%), en comparación con los generados en la fabricación de otros productos. Además, es el producto de mayor demanda diaria en la empresa. Las dimensiones y tolerancias del bloque samo \#4 se observan en la Fig. 2 y Tabla II, respectivamente.

TABLA I

DIMENSIONES DEL BLOQUE TIPO SAMO

\begin{tabular}{ccccc}
\hline \hline Bloque & $\begin{array}{c}\text { Largo } \\
{[\mathrm{cm}]}\end{array}$ & $\begin{array}{c}\text { Alto } \\
{[\mathrm{cm}]}\end{array}$ & $\begin{array}{c}\text { Ancho } \\
{[\mathrm{cm}]}\end{array}$ & $\begin{array}{c}\text { Espesor de Pared } \\
{[\mathrm{mm}]}\end{array}$ \\
\hline Samo \#3 & 40 & 20 & 7 & 13 \\
Samo \#4 & 40 & 20 & 9 & 11 \\
Samo \#5 & 40 & 20 & 12.5 & 11 \\
Samo \#6 & 40 & 20 & 15 & 11 \\
\hline \hline
\end{tabular}
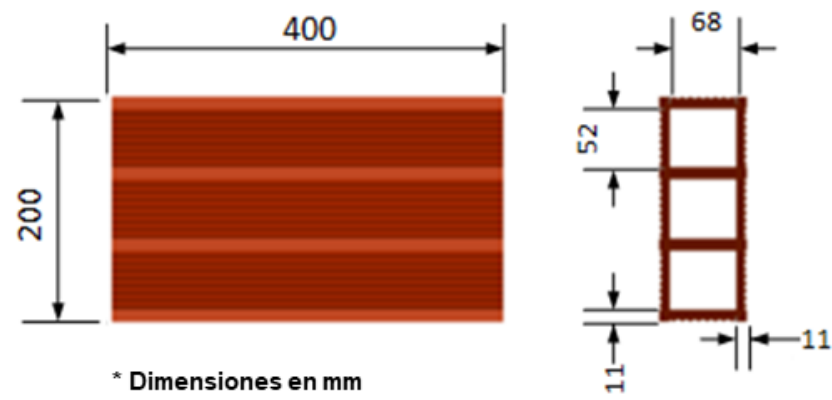

Fig. 2. Dimensiones del bloque samo \#4.

\section{Etapas de Fabricación de los Bloques}

En la Fig. 3, se muestran las etapas de fabricación del bloque samo \#4, a través de un diagrama de flujo. El proceso inicia con la extracción de su materia prima (arcilla) de la

TABLA II

DIMENSIONES Y TOLERANCIAS DEL BLOQUE SAMO \#4

\begin{tabular}{ccccl}
\hline \hline Bloque & $\begin{array}{c}\text { Largo } \\
{[\mathrm{cm}]}\end{array}$ & $\begin{array}{c}\text { Alto } \\
{[\mathrm{cm}]}\end{array}$ & $\begin{array}{c}\text { Ancho } \\
{[\mathrm{cm}]}\end{array}$ & $\begin{array}{c}\text { Espesor de Pared } \\
{[\mathrm{mm}]}\end{array}$ \\
\hline Valor Estándar & 40 & 20 & 9 & 11 \\
Valor Máximo & 41 & 20.5 & 9.2 & 13 \\
Valor Mínimo & 39 & 19.5 & 8.8 & 9 \\
\hline \hline
\end{tabular}

cantera, este proceso se realiza con la ayuda de maquinaria pesada. Luego de ser seleccionada y extraída, la arcilla es conducida a la planta donde se apila, para dar inicio al proceso de envejecimiento y maduración. Finalizada la etapa anterior, se mezcla la arcilla y la arena. Este procedimiento se realiza 
con la finalidad de garantizar una mezcla homogénea antes de ingresar por las tolvas de recepción de materia prima.

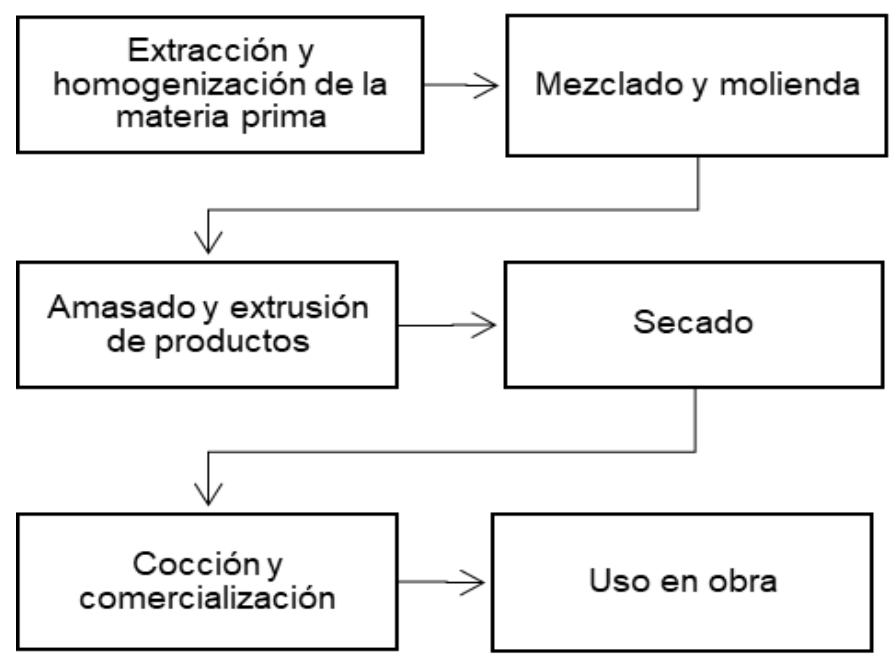

Fig. 3. Diagrama de flujo del proceso de fabricación del bloque.

Posterior al mezclado se lleva el material a la zona de molienda. En la Fig. 4, se muestra el molino tipo martillo de la planta, el cual, asegura la obtención de un porcentaje elevado de partículas finas en la mezcla. La importancia de tener un material más fino es que nos garantiza un producto con mejor acabado, mayor resistencia mecánica y con capacidad de humectarse más fácil y rápido, todo esto debido a la reducción en el tamaño de las partículas de arcilla [19].

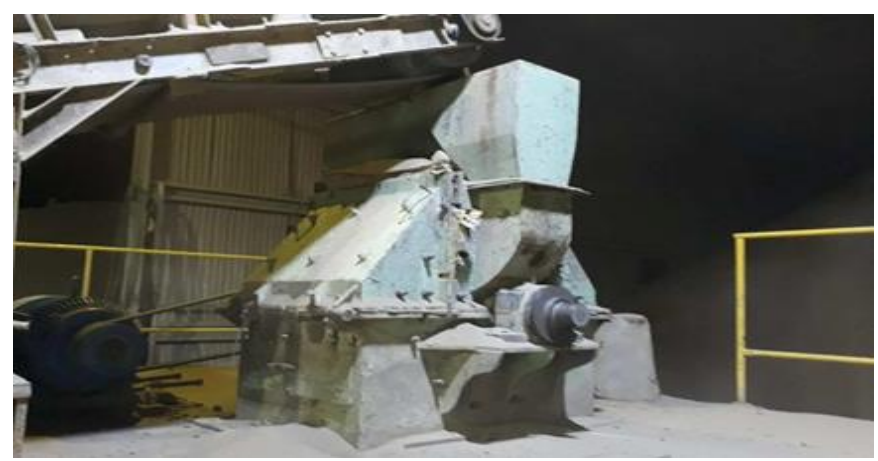

Fig. 4. Molino tipo martillo de la planta Gres Caribe S.A.

A continuación, el material molido entra a la planta con el propósito de ser extruido, cabe resaltar que para obtener buenos resultados en la extrusión debe tener un material lo más homogéneo posible, tanto en composición (arcilla y arena) como en su grado de humedad [20]. Para garantizar esto se usan máquinas como la amasadora y el laminador, las cuales se observan en la Fig. 5. En la amasadora es donde se genera la primera etapa de humectación del material, en ella se vierte un porcentaje no mayor a $40 \%$ del contenido de agua que tendrá el material al momento de ser extruido, el otro $60 \%$ se agrega a la mezcla en la bañera de la extrusora. El material es humectado y mezclado al mismo tiempo, esto se realiza con el propósito de que las partículas arcillosas formen capas mucho más rígidas. Una vez el material sale de la amasadora es enviado al laminador, el cual realiza un desmenuzado intensivo de las partículas más grandes que posea la mezcla y reduce el aire remanente en la pasta arcillosa. Finalmente, el material está listo para tomar forma y obtener la pieza cerámica.
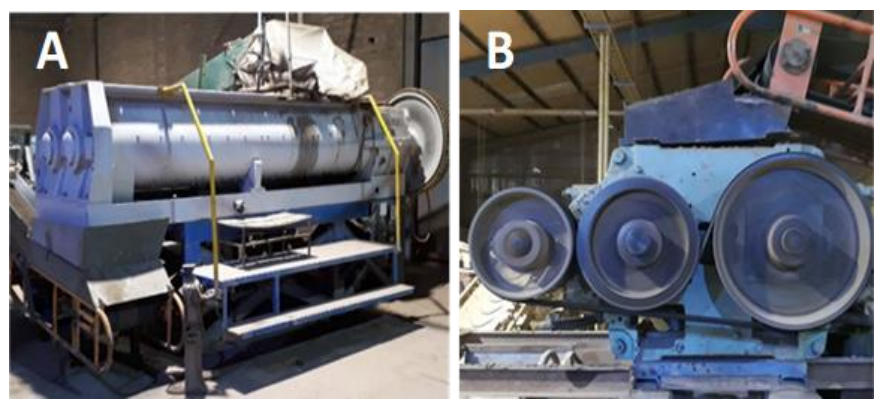

Fig. 5. Amasadora (A) y Laminador (B) de la planta Gres Caribe S.A.

La etapa de secado tiene como objetivo eliminar la mayor cantidad de agua que el producto ha ganado gracias a la fase de extrusión, esto para garantizar que el bloque llegue seco a la cocción y evitar que se estalle (choque térmico) por los altos contenidos de humedad. El túnel de secado maneja temperaturas entre 84 - $98{ }^{\circ} \mathrm{C}$. Esta temperatura se obtiene del calor residual del horno, el cual se extrae con la ayuda de un ventilador de tiro mixto, como se observa en la Fig. 6.

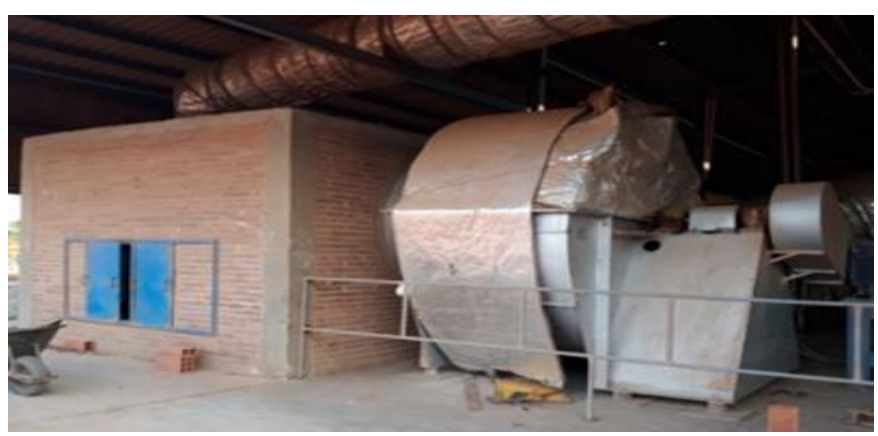

Fig. 6. Ventilador de tiro mixto del secadero en la planta Gres Caribe S.A.

La cocción es considerada la etapa más importante y delicada en la fabricación de los productos cerámicos, es en esta fase donde se le proporcionan las propiedades deseadas a la pieza. Durante la cocción, se transforman las propiedades iniciales de la arcilla, a través de un proceso llamado sinterización, dando origen a un cuerpo de alta resistencia mecánica, baja capacidad de absorción de agua, resistencia a la abrasión, de conductividad térmica baja entre otras propiedades [21]. En la planta de Gres Caribe S.A. los bloques tardan entre once y doce horas para completar el proceso, usando para su quema un horno túnel a gas natural y quemadores verticales. Una vez terminadas las etapas anteriores el producto sale ya cocido y listo para su comercialización, como se muestra en la Fig. 7. Cabe resaltar, que antes de la comercialización se realiza un control de calidad, donde el personal de planta selecciona el producto de primera, de segunda y el material dañado, el cual se conoce como el desperdicio del proceso. 


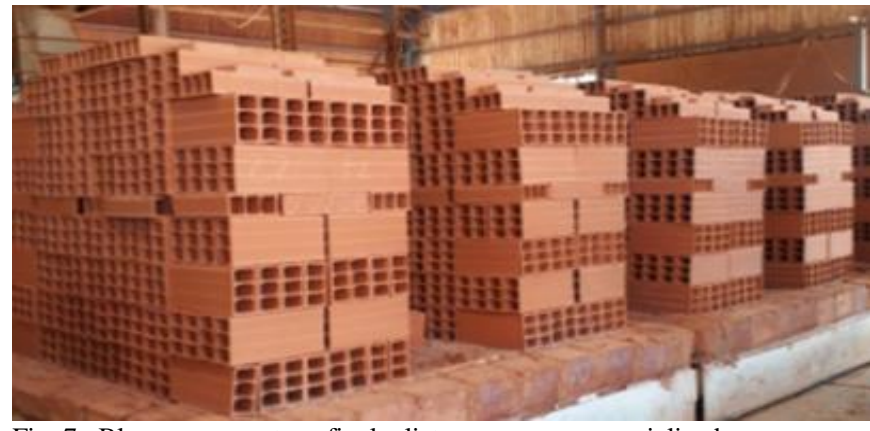

Fig. 7. Bloques en su etapa final y listos para ser comercializados.

\section{E. Pruebas de Campo Previas}

Ya conocidas las características de la materia prima y el proceso de fabricación del bloque, es posible realizar los experimentos pilotos. Estos se realizaron con la finalidad de conocer el comportamiento de la mezcla ante las variaciones en sus proporciones y, así, poder descartar las que no sean posibles de realizar. Todo el proceso de fabricación del bloque en las pruebas pilotos (preparación de la materia prima, extrusión, secado y cocción) fue realizado con supervisión de producción en las instalaciones de Gres Caribe S.A. Además, se determinó variar la arcilla entre $20 \%-80 \%$, la arena entre $10 \%-60 \%$ y el agua entre el 3\%-20\%. Cabe resaltar que en la selección de los intervalos no se tuvo en cuenta ninguna metodología estadística, solo se tuvo como referencia la mezcla utilizada por la empresa (60\% de arcilla, 30\% de arena y $10 \%$ de agua) y recomendaciones de producción en base a su experiencia. La materia prima utilizada se tomó de las pilas de homogenización y envejecimiento que están a pie de planta, verificando que para cada ensayo la arcilla tuviera el mismo tiempo de extracción envejecimiento, aproximadamente 6 meses. En la Tabla III, se muestran las proporciones establecidas para los experimentos pilotos.

TABLA III

MEZCLAS ESTABLECIDAS PARA LAS PRUEBAS DE CAMPO PREVIAS

\begin{tabular}{llll}
\multicolumn{4}{c}{ PRUEBAS DE CAMPO PREVIAS } \\
\hline \hline & $\begin{array}{c}\text { Arcilla } \\
{[\mathrm{kg}]}\end{array}$ & $\begin{array}{c}\text { Arena } \\
{[\mathrm{kg}]}\end{array}$ & $\begin{array}{c}\text { Agua } \\
{[\mathrm{kg}]}\end{array}$ \\
\hline 1 & 800 & 150 & 50 \\
2 & 600 & 300 & 100 \\
3 & 400 & 550 & 50 \\
4 & 200 & 600 & 200 \\
5 & 680 & 200 & 120 \\
6 & 450 & 450 & 100 \\
7 & 300 & 550 & 150 \\
8 & 550 & 350 & 100 \\
9 & 700 & 270 & 30 \\
10 & 350 & 610 & 40 \\
\hline \hline
\end{tabular}

Para la etapa de molienda del material se instalaron martillos nuevos en las pruebas realizadas con la finalidad de obtener una granulometría fina y constante. Cabe resaltar, que a medida que se desgastan los martillos aumenta el tamaño de las partículas de la mezcla, lo que conlleva a variaciones de las propiedades del producto final. En el laminador se controló el espacio entre rodillos para cada corrida, el cual fue de $5 \mathrm{~mm}$, con esto se logra mantener constantes las propiedades de la mezcla que ingresa a la extrusora.
Las mezclas fueron extruidas en días soleados, con el fin de garantizar la menor cantidad de humedad en la materia prima. El horario para la extrusión fue de 2:00 pm a 4:00 pm, por la disponibilidad de los equipos debido al cambio de turno. La presión de la cámara de vacío de la extrusora se mantuvo constante en 85 bar, con una velocidad de salida de 63 bloques por minuto. La corriente eléctrica consumida en la extrusión y la dureza del material recién extruido actualmente están fijados en 100 A y 14 bar respectivamente. Estos valores son dependientes del tipo de mezcla utilizada ya que, una mezcla con proporciones diferentes produciría variaciones en los valores de estos parámetros.

El secado del producto se realizó con un tiempo de permanencia de 36 horas a una temperatura de $94{ }^{\circ} \mathrm{C}$ controlada por un termostato. En la etapa de cocción se mantuvo constante el perfil de temperatura y el tiempo de permanencia en el horno. La temperatura en el horno es medida por termocuplas con un setpoint de $870{ }^{\circ} \mathrm{C}, 920^{\circ} \mathrm{C}$, $920^{\circ} \mathrm{C}$ y $900^{\circ} \mathrm{C}$ en los grupos de quemadores $1,2,3$ y 4 respectivamente. El tiempo de permanencia en el horno de los bloques es de 11 horas y 18 minutos.

Para controlar las cantidades de materia prima requeridas en cada corrida del experimento se depositan en sacos la arcilla y la arena, y luego se pesan en una báscula con certificado de calibración vigente propiedad de la empresa (cap. 2 ton y $1 \mathrm{~kg}$ de resolución). Después de completar el peso establecido para cada mezcla, se vierte el contenido de los sacos en la pala de un cargador, para realizar un mezclado previo antes de depositar el material en las tolvas de recepción de materia prima, donde inician el proceso de preparación para la extrusión. El agua es agregada en la mezcla en un $40 \%$ en la amasadora y el otro $60 \%$ se añade en la bañera de la extrusora; ésta se agrega por medio de una electroválvula instalada en la máquina, como se observa en la Fig. 8. Es importante determinar el caudal del agua que fluye hacia la mezcla, para así, poder relacionarlo con el tiempo de apertura de la electroválvula para obtener la cantidad de agua necesaria en cada corrida. Los cálculos se describen a continuación.

En primera instancia se cuantificó la cantidad de orificios de la tubería de suministro de agua en la amasadora, $\mathrm{N}_{1}$, y en la bañera de la extrusora, $\mathrm{N}_{2}$, obteniendo $\mathrm{N}_{1}=32$ y $\mathrm{N}_{2}=32$. Luego se midió el tiempo que le tomaba a la salida de agua a través del orificio el llenado de un volumen conocido, Vc. Se determinó que los tiempos de la amasadora, $\mathrm{t}_{1}$, $\mathrm{y}$ de la extrusora, $t_{2}$, eran iguales para un llenado de $350 \mathrm{ml}$, por ejemplo, lo cual ocurría en $\mathrm{t}_{1}=\mathrm{t}_{2}=60$ seg.
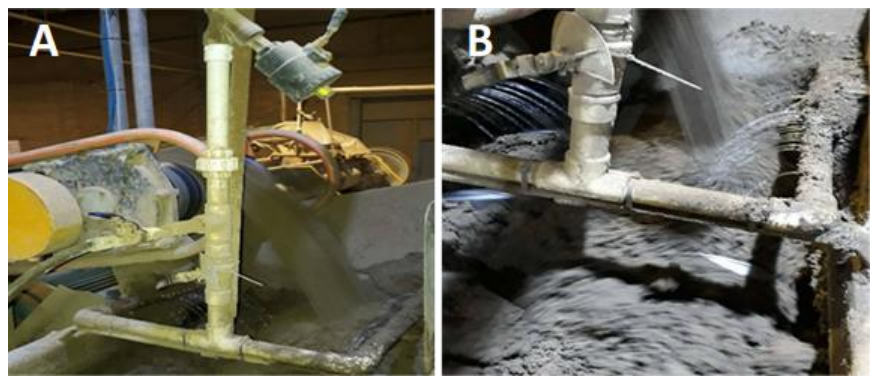
Fig. 8. Dosificación del agua en la amasadora (A) y en la bañera de extrusora (B).

A continuación, se determinó el caudal, Ca, que fluye desde uno de los orificios, mediante el uso de (1). Posteriormente se calcula el caudal de agua en la amasadora, C1, con (2), es decir, multiplicando por la cantidad de orificios presentes en cada instancia. Similarmente, se calcula el caudal en la bañera de la extrusora, C2, usando (3). Con los datos arriba presentados se obtiene $\mathrm{Ca}=0.35 \mathrm{lt} / \mathrm{min}, \mathrm{C}_{1}=11.2 \mathrm{lt} / \mathrm{min}$, $\mathrm{C}_{2}=11.2 \mathrm{lt} / \mathrm{min}$.

$$
\begin{aligned}
& \mathrm{C}_{\mathrm{a}}=\frac{\mathrm{v}_{\mathrm{C}}}{\mathrm{t}} \\
& \mathrm{C}_{1}=\mathrm{C}_{\mathrm{a}} \mathrm{N}_{1} \\
& \mathrm{C}_{2}=\mathrm{C}_{\mathrm{a}} \mathrm{N}_{2}
\end{aligned}
$$

La unidad de medida para la dosificación de las materias primas es el peso, por lo cual, se debe relacionar el caudal de agua, con la magnitud en peso de la misma. Para esto se determinó el volumen de agua solicitado en cada corrida (Vs) a través de (4), donde $d_{\mathrm{H} 2 \mathrm{O}}=1000 \mathrm{~kg} / \mathrm{m} 3$. Finalmente se calcula el tiempo de apertura de la electroválvula para obtener la cantidad de agua deseada en la amasadora, $t_{3}$, $y$ en la bañera de la extrusora, $\mathrm{t}_{4}$, utilizando (5) y (6), respectivamente.

$$
\begin{aligned}
& \mathrm{V}_{\mathrm{s}}=\frac{\mathrm{m}}{\mathrm{d}_{\mathrm{H}_{2} \mathrm{O}}} \\
& \mathrm{t}_{3}=\frac{0,4 \mathrm{~V}_{\mathrm{s}}}{\mathrm{C}_{1}} \\
& \mathrm{t}_{4}=\frac{0,6 \mathrm{~V}_{\mathrm{s}}}{\mathrm{C}_{2}}
\end{aligned}
$$

\section{F. Resultados de las Pruebas de Campo Previas}

Las pruebas de campo realizadas evidenciaron importantes variaciones de propiedades, tanto en la fabricación del bloque como en el producto terminado. Cabe resaltar, que solo se realizaron pruebas cualitativas en esta fase, los ensayos de compresión y absorción de agua serán objeto de los bloques fabricados bajo la metodología del diseño de experimento con mezclas. Las observaciones más relevantes se describen a continuación.

Los bloques que se fabricaron con un porcentaje de arcilla comprendido entre $20 \%$ y $45 \%$, presentaron problemas de plasticidad lo que perjudicó la etapa de moldeo. Además, los bloques crudos eran muy quebradizos y no tenían la cohesión necesaria para resistir esfuerzos. Otra característica relevante fue que gran parte de la producción presentó un color rojo opaco debido a que la arcilla contiene óxido férrico $\left(\mathrm{Fe}_{2} \mathrm{O}_{3}\right)$ que es el que le confiere al bloque su color rojo característico. En la Fig. 9, se muestran algunos de los bloques fabricados con bajo contenido de arcilla.

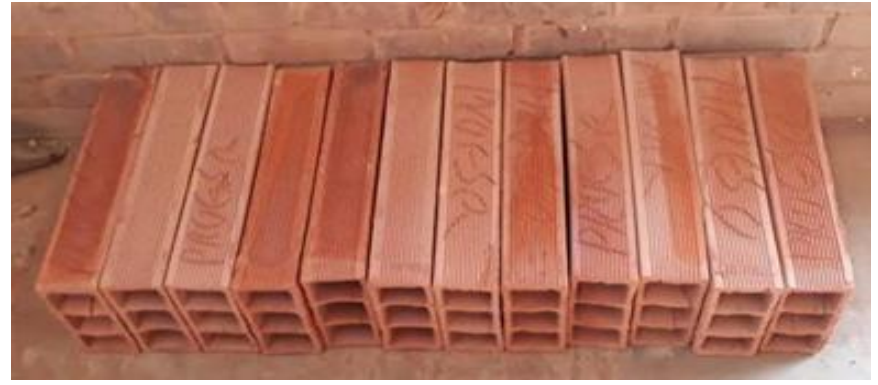

Fig. 9. Bloques con bajo contenido de arcilla.

Los bloques con un porcentaje de arcilla mayor a $60 \%$ presentaron exceso de plasticidad, lo que dificultó la etapa de moldeo por adhesión en el dado de extrusión. Además, estos bloques fundían a una temperatura más elevada que lo acostumbrado, lo que eleva los costos de producción en la etapa de cocción, por la mayor permanencia de tiempo en el horno y por ende el gas natural consumido. En la Fig. 10, se observa un bloque fabricado con alto contenido de arcilla.

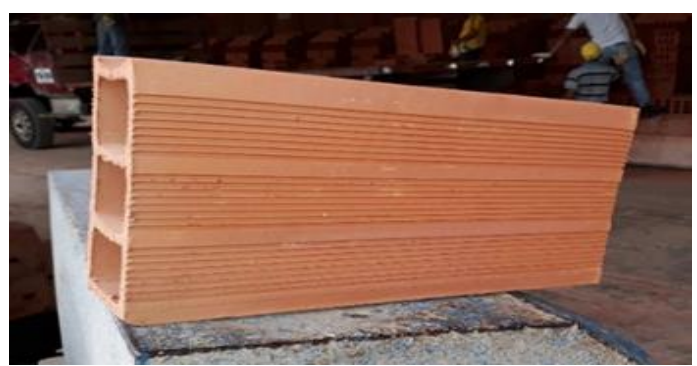

Fig. 10. Bloques con alto contenido de arcilla.

Los bloques con un porcentaje de arena comprendido entre $10 \%$ y $30 \%$, presentaron problemas de moldeo por adhesión en el dado de extrusión. Además, la arena conforma el esqueleto resistente que soporta las cargas y evita la fisuración del bloque, por lo tanto, los bloques con bajos porcentajes de arena exhibían alta contracción en la quema, lo que desencadenaba en grietas en el bloque. En la Fig. 11, se muestran algunos de los bloques fabricados con bajo contenido de arena.
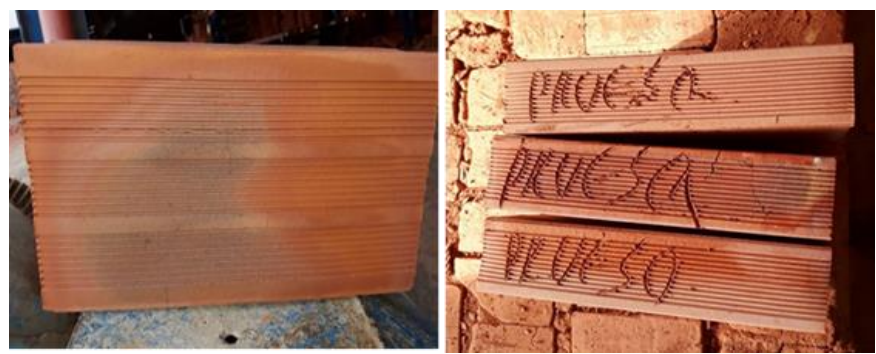

Fig. 11. Bloques con bajo contenido de arena.

Los bloques con un porcentaje de arena mayor a $45 \%$ presentaron alta porosidad, y por ende baja resistencia mecánica. Además, se obtuvieron tiempos de secado demasiado rápidos, lo que conllevo al agrietamiento del producto por contracción. El alto contenido de arena bajó en exceso la plasticidad del producto, por lo que presentaba problemas de moldeo y fragilidad en etapa cruda. En la Fig. 12, se muestran algunos de los bloques fabricados con alto 
contenido de arena.
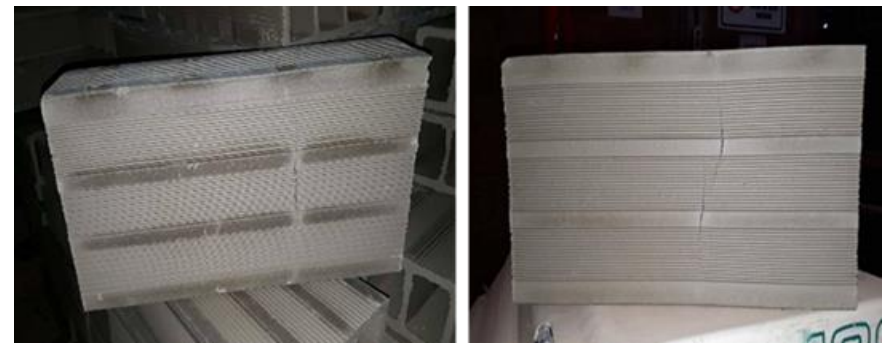

Fig. 12. Bloques con alto contenido de arena.

Las mezclas con un porcentaje de agua menor al $5 \%$ presentaron problemas de moldeo, lo que se refleja en sobreesfuerzos de la maquinaria, resultando en mayores gastos energéticos y de repuestos por desgaste excesivo de las piezas. Además, en la corrida 9 se produjo una presión interna de trabajo muy elevada, lo que generó un desperfecto en el dado de extrusión, como se observa en la Fig. 13, esto debido a que se ensayó la mezcla con el porcentaje de agua más bajo establecido (3\%).

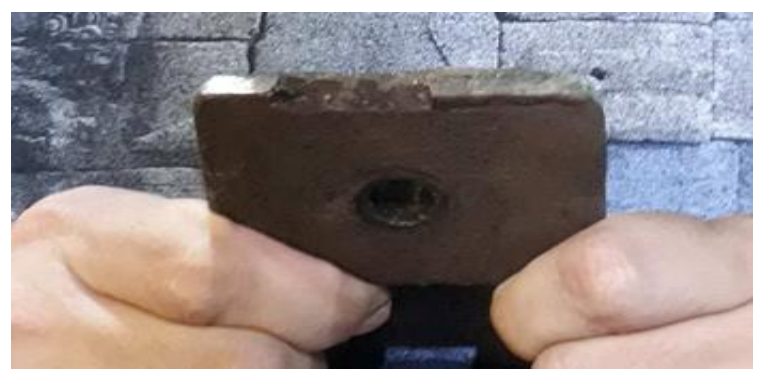

Fig. 13. Desperfecto en dado de extrusión.

Cuando se agregó a la mezcla un porcentaje de agua mayor a $12 \%$, esta fluyó más rápido por la extrusora, pero se obtenía un bloque con largos tiempos de secado lo que retrasaba la producción y aumentaba los costos de fabricación. Además, cuando el porcentaje de agua era excesivamente alto, cercanos al $20 \%$, se producía el "erizo" de la mezcla, como se muestra en la Fig. 14.
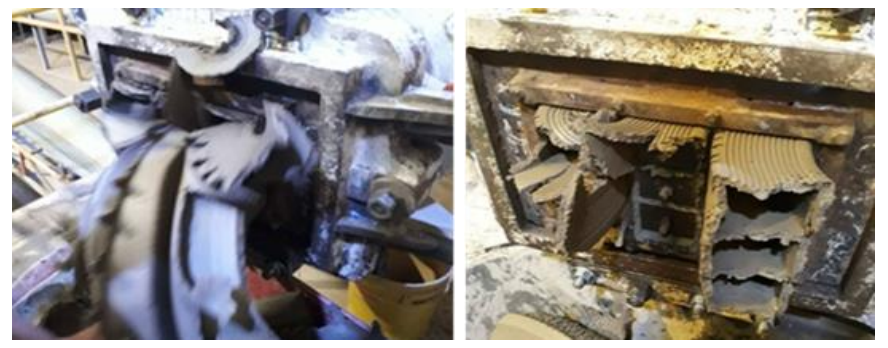

Fig. 14. "Erizo" de la mezcla en bloques con alto contenido de agua.

En el secado se generaron grietas y se hizo difícil controlar las dimensiones del bloque por la evaporación del agua agregada. En ocasiones el agua retirada en el secadero no fue suficiente e ingresaban al horno bloques con alto contenido de humedad, lo que producía la fractura del producto debido al choque térmico, como se observa en la Fig. 15.

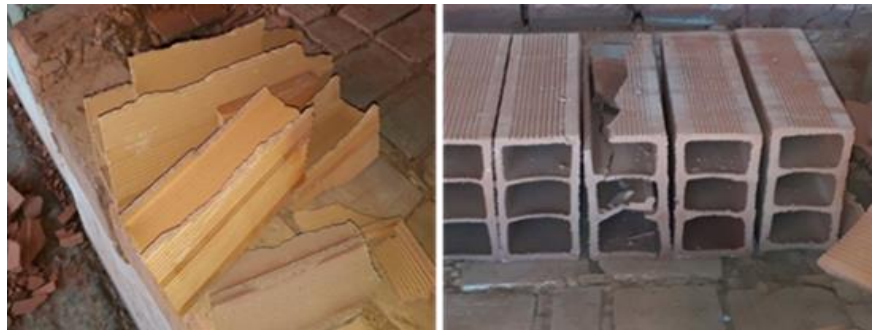

Fig. 15. Choque térmico por alto contenido de humedad en los bloques.

\section{DISEÑO DEL EXPERIMENTO DE MEZCLAS}

El diseño de experimentos es una metodología desarrollada para aplicar sistemáticamente la estadística a la experimentación. Consiste en determinar un cierto número de pruebas que se deben realizar, para adquirir datos, que al ser analizados estadísticamente permiten obtener conclusiones válidas y objetivas a los interrogantes planteados [1].

El diseño de experimentos tiene como objetivo generar conocimiento acerca de un proceso en el transcurso de la experimentación. Por tanto, se implementa efectivamente para aquellos procesos, que su rendimiento se ve afectado por varios factores. Un diseño experimental exitoso debe cumplir con las etapas de planeación, realización, análisis, interpretación, control y conclusiones finales; siendo la planeación la etapa más importante y por tanto a la que se le debe dedicar más atención y tiempo [9].

\section{A. Conceptos Básicos}

Experimento. Se define como un conjunto particular de circunstancias, que se encuentran bajo un protocolo específico para observar y evaluar, las implicaciones que tienen los cambios en las condiciones de operación de un sistema o proceso, sobre las propiedades del producto o resultado [3].

Factores. Son las variables que se investigan en el experimento y tienen influencia en las variables de respuesta estudiadas. Los factores pueden ser controlables o no controlables, estos se reflejan en la media y en la variabilidad de la respuesta [9].

Niveles y tratamientos. Los niveles se definen como los diferentes valores reales que se le asignan a cada factor en la realización del experimento. La combinación de niveles de todos los factores estudiados se denomina tratamiento, en el caso de experimentar con un solo factor, cada nivel es un tratamiento [22].

\section{B. Diseño de Experimentos con Mezclas}

En el diseño de experimentos de mezclas los factores son los componentes de una mezcla. Las variables de respuesta dependen de las proporciones con que participan los componentes de la mezcla y no de la cantidad total de la misma. En un diseño de experimentos con mezclas se tendrán $\mathrm{q}$ componentes y cada tratamiento consiste en una combinación de dichos componentes. Sí $\mathrm{X}_{1}, \mathrm{X}_{2}, \ldots, \mathrm{Xq}$ son las variables que representan las proporciones de los componentes de la mezcla, los valores de $\mathrm{Xi}$ deben cumplir las restricciones dadas en (7) y (8). 


$$
\begin{gathered}
0 \leq \mathrm{X}_{\mathrm{i}} \leq 1 \\
\sum_{\mathrm{i}=1}^{\mathrm{q}} \mathrm{X}_{\mathrm{i}}=\mathrm{X}_{1}+\mathrm{X}_{2}+\cdots+\mathrm{X}_{\mathrm{q}}=1
\end{gathered}
$$

La primera restricción indica que los valores de las proporciones deben ser cantidades entre 0 y 1 , y la segunda restricción ocasiona que las q proporciones sumen la unidad, esto significa que los componentes no pueden ser manipulados independientemente unos de otros [4]. Debido a esto, los diseños de experimentos con mezclas se diferencian de los diseños factoriales y de los diseños de superficie de respuesta. Las regiones experimentales de los diseños de mezclas se representan mediante simplex, estas son figuras geométricas que permiten ilustrar q componentes en q-1 dimensiones. En la Fig. 16, se muestra la representación de los diseños de mezclas para $\mathrm{q}=2$ y $\mathrm{q}=3$.

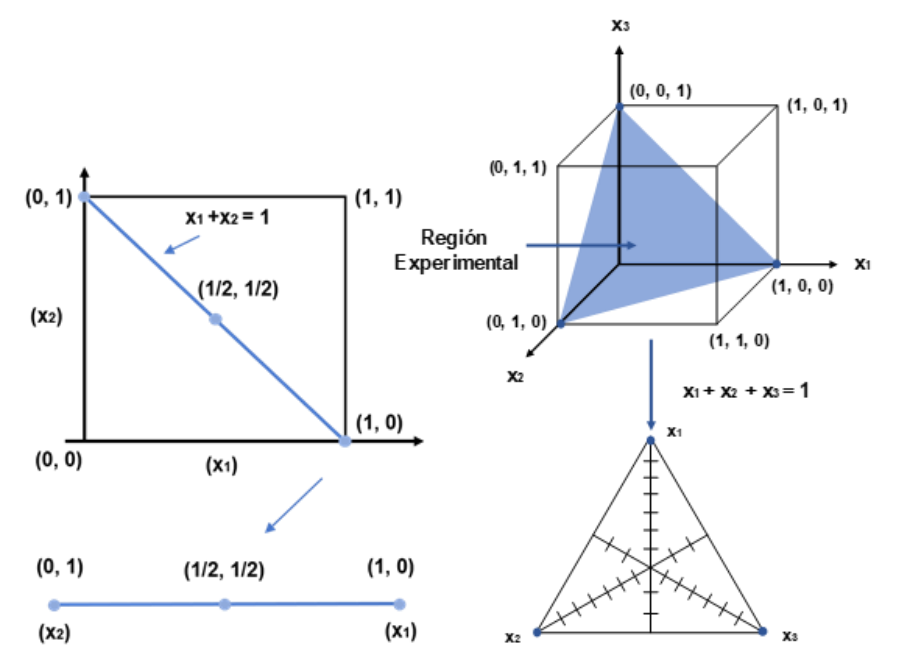

A

B

Fig. 16. Representación geométrica de los diseños experimentales de mezclas con $q=2$ (A) y $q=3$ (B).

\section{1) Diseños simplex}

Los diseños de tipo simplex se usan para estudiar el efecto que tienen los elementos de la mezcla sobre las variables de respuesta. Los dos tipos básicos de diseños son el simplexlattice y el simplex con centroide. El diseño simplex-lattice $\{q$, $\mathrm{m}$ ) consta de q componentes y permite ajustar un modelo estadístico de orden $\mathrm{m}$. Las proporciones asumidas por cada componente toman los $\mathrm{m}+1$ valores entre 0 y 1 dados por (9).

$$
\mathrm{X}_{\mathrm{i}}=0,1 / \mathrm{m}, 2 / \mathrm{m}, \ldots, \mathrm{m} / \mathrm{m}
$$

El diseño simplex con centroide se aplica con pocos componentes, consiste en $2 q-1$ puntos definidos de la siguiente forma: las q mezclas puras, todos los puntos medios de las aristas definidas por cada dos vértices del simplex, los centroides de las caras definidas por cada tres vértices del simplex y así hasta obtener el centroide global [2].

\section{2) Diseño de mezclas con restricciones}

Las restricciones a los componentes modifican la forma de la región experimental, resultando en la mayoría de los casos un polígono o hiperpolígono irregular, que imposibilita usar los modelos del tipo simplex, por lo que se debe recurrir a otros criterios considerados usualmente en sistemas computacionales [13].

Los vértices extremos es una metodología propuesta por McLean y Anderson (1966) para estudiar mezclas con restricciones superiores e inferiores en sus componentes. Esta propone usar los vértices de la región experimental como base del diseño, junto con una serie de puntos a lo largo de los centroides de la subregión y el centroide global [13].

El D-óptimo es un criterio utilizado para seleccionar los puntos del diseño de una lista de puntos candidatos suministrada por el experimentador. Estos son seleccionados de tal manera que las varianzas de los coeficientes de regresión en el modelo con mezclas son minimizadas. La efectividad del criterio depende de la lista de puntos candidatos que sea suministrada, el ajuste del modelo estadístico propuesto y de la cantidad de puntos del diseño a ser seleccionados [13].

\section{Selección del Diseño de Mezclas}

En base a los resultados de los experimentos pilotos, además de la literatura consultada y de la información suministrada por el departamento de producción, en relación de los porcentajes de mezcla utilizados típicamente para el bloque samo \#4, se determinó utilizar un diseño de mezclas con restricciones ya que las materias primas utilizadas no permiten explorar las mezclas puras, necesarias para los modelos del tipo simplex. El diseño D-óptimo se ajusta a esta situación; además, de ser un criterio superior a los vértices extremos [13].

\section{Selección de los Componentes de la Mezcla y de las Variables de Salida}

Para realizar la mezcla del bloque samo \#4, se utilizarán 3 componentes como variables de entrada, los cuales son arcilla $\left(\mathrm{X}_{1}\right)$, arena $\left(\mathrm{X}_{2}\right)$ y agua $\left(\mathrm{X}_{3}\right)$. Las materias primas serán suministradas por Gres Caribe S.A. para garantizar mayor precisión en los experimentos. Las variables de respuesta serán la resistencia a la compresión $\left(\mathrm{Y}_{1}\right)$ y el porcentaje de absorción de agua fría $\left(\mathrm{Y}_{2}\right)$ y por ebullición $\left(\mathrm{Y}_{3}\right)$.

\section{E. Selección de los Límites Superior e Inferior de cada Componente}


Los límites superior e inferior de cada componente se obtuvieron a partir del análisis de resultados de los experimentos pilotos, además se tuvo en cuenta el porcentaje de cada componente utilizado actualmente y los datos suministrados por la empresa. Los límites de las restricciones y la naturaleza de las mismas se muestran en la Tabla IV.

TABLA IV

RESTRICCIONES PARA LOS COMPONENTES DE LA MEZCLA

\begin{tabular}{|c|c|c|}
\hline Componentes & $\begin{array}{c}\text { Límite } \\
\text { Inferior } \\
\text { /superior }\end{array}$ & Motivo de la Restricción \\
\hline Arcilla & 0.45 & $\begin{array}{l}\text { La arcilla contribuye con plasticidad a la } \\
\text { mezcla, esta propiedad se debe a que el } \\
\text { agua envuelve las partículas laminares } \\
\text { produciendo un efecto lubricante que } \\
\text { facilita el deslizamiento de unas partículas } \\
\text { sobre otras cuando se ejerce un esfuerzo } \\
\text { sobre ellas, por lo tanto, en menores } \\
\text { proporciones se pierde cohesión en la } \\
\text { mezcla y no se obtiene un producto con la } \\
\text { resistencia deseada. }\end{array}$ \\
\hline
\end{tabular}

0.60 En cantidades elevadas, el óxido férrico $\left(\mathrm{Fe}_{2} \mathrm{O}_{3}\right)$ presente en la arcilla puede ocasionar altas temperaturas de fundición para el bloque, lo que elevaría los costos en la cocción, además, se pierde resistencia en el bloque. La arcilla eleva la plasticidad de la mezcla, lo que en exceso produciría problemas por adhesión en el proceso de moldeo.

Arena $\quad 0.3 \quad$ Debido a su bajo índice de plasticidad, la arena compensa el exceso de plasticidad en la mezcla, por lo tanto, sirve como material estabilizante que ayuda al moldeo del bloque. Además, ayuda a obtener un bloque ligero y a reducir la contracción en la cocción.

0.45 Por su baja plasticidad, en cantidades elevadas perjudicaría el moldeo adecuado del bloque, a su vez se produciría un bloque poroso lo que reduciría la resistencia del mismo.

Agua $\quad 0.05 \quad$ En menores proporciones, el producto saldría más duro de la extrusión y con menor velocidad, lo que se traduce en un mayor gasto energético y mayor desgaste en las piezas de la maquinaria pues se aumenta la presión interna. Adicional a esto su secado sería rápido lo que ocasionaría una contracción severa del bloque y, por lo tanto, eleva el riesgo de grietas.

0.12 Si se aumenta sensiblemente el porcentaje, la cohesión desciende rápidamente, facilitando el deslizamiento entre partícula y partícula, con lo cual la masa tendría a fluir con mayor velocidad, pero se obtendría una mezcla con un tiempo de secado prolongado. Por lo tanto, se produciría un producto difícil de controlar dimensionalmente y que puede llegar a zona de cocción con una alta humedad que ocasione la fractura del producto debido al choque térmico.

\section{F. Diseño de Mezclas Usando el Criterio D-Óptimo}

Se escogió el criterio D-óptimo como metodología para la selección de los puntos experimentales. Teniendo en cuenta las restricciones para cada variable establecidas, se procede a determinar la región experimental del bloque samo \#4. En la Fig. 17 se ilustra la región experimental factible para el bloque, la cual es un polígono irregular de seis vértices lo que impide la utilización de diseños de tipo simplex y confirma el uso del diseño D-óptimo para el experimento.

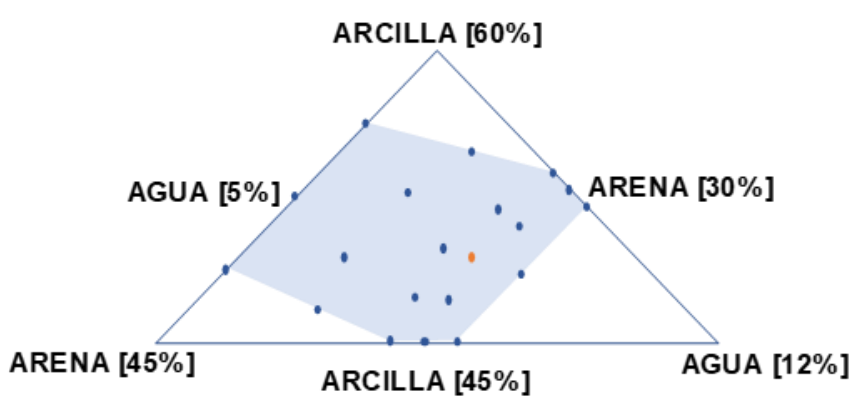

Fig. 17. Región experimental bloque samo \# 4.

Se estableció un experimento de $\mathrm{q}=3$ componentes $\left(\mathrm{X}_{1}=\right.$ Arcilla, $\mathrm{X}_{2}=$ Arena, $\mathrm{X}_{3}=$ Agua $)$ con tres respuestas $(\mathrm{Y} 1=$ Resistencia a la compresión, Y2 = Porcentaje de absorción de agua fría, $\mathrm{Y} 3$ = Porcentaje de absorción de agua por ebullición). La región experimental está delimitada por las siguientes restricciones, $0.45 \leq \mathrm{X}_{1} \leq 0.60 ; 0.30 \leq \mathrm{X}_{2} \leq 0.45$; $0.05 \leq X_{3} \leq 0.12$. En cada corrida serán extruidas a velocidad constante 1 ton $(1000 \mathrm{~kg})$ por mezcla, por lo cual, la suma de la proporción de cada componente debe ser igual a esa cantidad. El modelo estadístico ajustado para el experimento fue el cúbico completo. Inicialmente se utilizará el modelo de mayor grado posible, pero luego se determinará a través de los criterios valor-p y coeficientes de determinación (R2 y R2ajus), si este modelo es adecuado o si es suficiente con un modelo de menor grado para explicar el comportamiento de cada respuesta. Para mezclas de tres componentes, como es el caso del bloque samo \#4, el modelo canónico de tercer orden está dado por (10).

$$
\begin{gathered}
\mathrm{E}(\mathrm{Y})=\beta_{1} \mathrm{X}_{1}+\beta_{2} \mathrm{X}_{2}+\beta_{3} \mathrm{X}+\beta_{12} \mathrm{X}_{1} \mathrm{X}_{2}+\beta_{13} \mathrm{X}_{1} \mathrm{X}_{3}+ \\
\beta_{23} \mathrm{X}_{2} \mathrm{X}_{3}+\beta_{123} \mathrm{X}_{1} \mathrm{X}_{2} \mathrm{X}_{3}+\delta_{12} \mathrm{X}_{1} \mathrm{X}_{2}\left(\mathrm{X}_{1}-\mathrm{X}_{2}\right)+ \\
\delta_{13} \mathrm{X}_{1} \mathrm{X}_{3}\left(\mathrm{X}_{1}-\mathrm{X}_{3}\right)+\delta_{23} \mathrm{X}_{2} \mathrm{X}_{3}\left(\mathrm{X}_{2}-\mathrm{X}_{3}\right)
\end{gathered}
$$

Los puntos candidatos considerados teniendo en cuenta el modelo de regresión escogido fueron: los vértices de la región, las mezclas de comprobación axiales en el centro de la línea que conecta los vértices con el centroide de la región viable, los centroides de los bordes conectando cada par de vértices adyacentes y el centroide global del diseño. En total son veinte corridas para el experimento. Las corridas se duplicaron con la finalidad de que el software seleccione, a través del criterio Dóptimo, los veinte puntos del diseño de una lista de cuarenta corridas posibles, y así poder garantizar réplicas que permitan estimar el error experimental y verificar la adecuación del 
modelo. Estas variables se introducen al software de análisis estadístico Statgraphics Centurion XVI, con el fin de obtener los puntos del diseño. En la Tabla $\mathrm{V}$ se muestran los puntos experimentales seleccionados por el software y, por lo tanto, cumplieron con el criterio de optimización.

TABLA V

Lista de PUNTOS CANDIDATOS DE

STATGRAPHICS CENTURION XVI

\begin{tabular}{|c|c|c|c|c|}
\hline & & $\begin{array}{c}\text { Arcilla } \\
{[\mathrm{kg}]}\end{array}$ & $\begin{array}{c}\text { Arena } \\
{[\mathrm{kg}]}\end{array}$ & $\begin{array}{c}\text { Agua } \\
{[\mathrm{kg}]}\end{array}$ \\
\hline$*$ & 1 & 600 & 350 & 50 \\
\hline * & 2 & 600 & 300 & 100 \\
\hline * & 3 & 500 & 450 & 50 \\
\hline$*$ & 4 & 450 & 450 & 100 \\
\hline * & 5 & 580 & 300 & 120 \\
\hline * & 6 & 450 & 430 & 120 \\
\hline \multirow[t]{10}{*}{ * } & 7 & 565 & 365 & 70 \\
\hline & 8 & 565 & 340 & 95 \\
\hline & 9 & 515 & 415 & 70 \\
\hline & 10 & 490 & 415 & 95 \\
\hline & 11 & 555 & 340 & 105 \\
\hline & 12 & 490 & 405 & 105 \\
\hline & 13 & 600 & 325 & 75 \\
\hline & 14 & 550 & 400 & 50 \\
\hline & 15 & 525 & 375 & 100 \\
\hline & 16 & 590 & 300 & 110 \\
\hline \multirow[t]{2}{*}{ * } & 17 & 475 & 450 & 75 \\
\hline & 18 & 450 & 440 & 110 \\
\hline \multirow[t]{2}{*}{$*$} & 19 & 515 & 365 & 120 \\
\hline & 20 & 530 & 380 & 90 \\
\hline$*$ & 21 & 600 & 350 & 50 \\
\hline * & 22 & 600 & 300 & 100 \\
\hline * & 23 & 500 & 450 & 50 \\
\hline$*$ & 24 & 450 & 450 & 100 \\
\hline * & 25 & 580 & 300 & 120 \\
\hline \multirow[t]{3}{*}{$*$} & 26 & 450 & 430 & 120 \\
\hline & 27 & 565 & 365 & 70 \\
\hline & 28 & 565 & 340 & 95 \\
\hline \multirow[t]{2}{*}{$*$} & 29 & 515 & 415 & 70 \\
\hline & 30 & 490 & 415 & 95 \\
\hline$*$ & 31 & 555 & 340 & 105 \\
\hline * & 32 & 490 & 405 & 105 \\
\hline$*$ & 33 & 600 & 325 & 75 \\
\hline \multirow[t]{7}{*}{$*$} & 34 & 550 & 400 & 50 \\
\hline & 35 & 525 & 375 & 100 \\
\hline & 36 & 590 & 300 & 110 \\
\hline & 37 & 475 & 450 & 75 \\
\hline & 38 & 450 & 440 & 110 \\
\hline & 39 & 515 & 365 & 120 \\
\hline & 40 & 530 & 380 & 90 \\
\hline
\end{tabular}

* Puntos experimentales seleccionados por el software.

Los puntos experimentales seleccionados fueron los seis vértices, todos se corren dos veces (\#1 - \#6 y \#21 - \#26). Los centros de borde también se corren (\#17, \#19, \#33 y \#34), al igual que las mezclas de comprobación axial (\#7, \#29, \#31 y \#32). No fue seleccionado el centroide global de la región.

\section{REALIZACIÓN DEL EXPERIMENTO}

El experimento de mezclas para el bloque samo \#4 fue realizado teniendo en cuenta las mismas consideraciones de las pruebas previas, en cuanto a variables del proceso. Las respuestas fueron obtenidas a través de ensayos realizados bajo la norma NTC 4017 (métodos para muestreo y ensayo de unidades de mampostería y otros productos de arcilla). Los ensayos de resistencia a la compresión en los bloques fueron realizados por la empresa Concrelab SAS y los ensayos de absorción de agua fría y por ebullición se realizaron en el laboratorio de Gres Caribe S.A.

\section{A. Resultados de los Experimentos}

TABLA VI

Resultados ObTenidos en CADA Punto EXPERIMENTAL

\begin{tabular}{lllllll}
\hline \hline & $\begin{array}{c}\text { Arcilla } \\
{[\mathrm{kg}]}\end{array}$ & $\begin{array}{c}\text { Arena } \\
{[\mathrm{kg}]}\end{array}$ & $\begin{array}{c}\text { Agua } \\
{[\mathrm{kg}]}\end{array}$ & $\begin{array}{c}\text { Res. } \\
\text { Comp. } \\
{\left[\mathrm{kgf} / \mathrm{cm}^{2}\right]}\end{array}$ & $\begin{array}{c}\text { Abs. } \\
\text { Agua } \\
\text { Fría }[\%]\end{array}$ & $\begin{array}{c}\text { Abs. por } \\
\text { Ebullición } \\
{[\%]}\end{array}$ \\
\hline 1 & 600 & 350 & 50 & 55.43 & 14.65 & 18.01 \\
2 & 600 & 300 & 100 & 57.66 & 14.96 & 16.84 \\
3 & 500 & 450 & 50 & 30.74 & 17.43 & 20.91 \\
4 & 450 & 450 & 100 & 22.94 & 22.74 & 26.76 \\
5 & 580 & 300 & 120 & 50.83 & 16.89 & 17.92 \\
6 & 450 & 430 & 120 & 28.51 & 24.07 & 23.93 \\
7 & 565 & 365 & 70 & 35.12 & 16.24 & 19.05 \\
8 & 475 & 450 & 75 & 31.72 & 20.72 & 24.97 \\
9 & 515 & 365 & 120 & 24.8 & 16.33 & 17.58 \\
10 & 600 & 350 & 50 & 63.93 & 15.21 & 23.56 \\
11 & 600 & 300 & 100 & 52.71 & 14.96 & 19.03 \\
12 & 500 & 450 & 50 & 29.32 & 20.09 & 24.23 \\
13 & 450 & 450 & 100 & 28.03 & 23.84 & 25.62 \\
14 & 580 & 300 & 120 & 48.69 & 13.74 & 16.36 \\
15 & 450 & 430 & 120 & 33.18 & 19.45 & 25.94 \\
16 & 515 & 415 & 70 & 30.64 & 16.44 & 19.21 \\
17 & 555 & 340 & 105 & 35.04 & 15.79 & 16.05 \\
18 & 490 & 405 & 105 & 31.85 & 17.93 & 22.53 \\
19 & 600 & 325 & 75 & 60.94 & 12.52 & 18.47 \\
20 & 550 & 400 & 50 & 37.63 & 20.36 & 21.84 \\
\hline \hline
\end{tabular}

Las respuestas obtenidas con los puntos experimentales que cumplieron con el criterio de optimización se muestran en la Tabla VI.

\section{B. Ajuste del Modelo y Caracterización de la Superficie de Respuesta}

Para estimar los coeficientes de los modelos estadísticos, se usó Statgraphics Centurion XVI, el cual aplica el principio de mínimos cuadrados. Preliminarmente se estableció un modelo de regresión cúbico completo para el experimento, pero se debe verificar si este modelo explica correctamente las respuestas o si es suficiente con uno de menor grado.

\section{1) Resistencia a la compresión}

Para la resistencia a la compresión se obtuvo la Tabla VII donde se muestra el análisis entre los modelos de regresión. En esta se aprecia el valor-P, el cual prueba si el modelo es estadísticamente significativo cuando se le compara con el cuadrado medio del término de abajo. Normalmente, se seleccionaría un modelo con un valor-P menor que 0.05 , asumiendo que se trabaja al nivel de confianza del 95\% [23]. Teniendo en cuenta este criterio se escogería al modelo lineal, pero el valor-P también es menor que 0.05 en el modelo cuadrático, por lo tanto, también podría tenerse en cuenta. 
TABLA VII

ANÁLISIS ENTRE LOS MODELOS ESTADÍSTICOS PARA LA RESISTENCIA A LA COMPRESIÓN

\begin{tabular}{llllll}
\hline \hline Fuente & $\begin{array}{c}\text { Suma de } \\
\text { Cuadrados }\end{array}$ & Gl & $\begin{array}{c}\text { Cuadrado } \\
\text { Medio }\end{array}$ & Razón-F & Valor-P \\
\hline Media & 31182.1 & 1 & 31182.1 & & \\
Lineal & 2448.09 & 2 & 1224.04 & 26.60 & 0.0000 \\
Cuadrático & 534.821 & 3 & 178.274 & 10.08 & 0.0008 \\
Cúbico & 4.67292 & 1 & 4.67292 & 0.25 & 0.6254 \\
Especial & & & & & \\
Cúbico & 73.6168 & 3 & 24.5389 & 1.45 & 0.2864 \\
Error & 169.276 & 10 & 16.9276 & & \\
Total & 34412.6 & 20 & & & \\
\hline \hline
\end{tabular}

Para la selección del modelo de regresión además del valor$\mathrm{P}$, se tiene el criterio de los coeficientes de determinación ( $\mathrm{R}$ Cuadrada y R-Cuadrada ajd.) expuestos en la Tabla VIII, los cuales muestran el porcentaje de la variación de la respuesta que es explicada por el correspondiente modelo [24]. En este caso, el modelo cuadrático es el indicado, ya que maximiza la R-Cuadrada ajd. En la Tabla IX se muestra el análisis de varianza para el modelo cuadrático completo, junto con la estimación de sus coeficientes.

TABLA VIII

ANÁLISIS DE COEFICIENTES DE DETERMINACIÓN PARA LA

\begin{tabular}{lccc}
\multicolumn{3}{c}{ RESISTENCIA A LA COMPRESIÓN } \\
\hline \hline \multicolumn{1}{c}{ Modelo } & ES & $\begin{array}{c}\text { R- } \\
\text { Cuadrada }\end{array}$ & $\begin{array}{c}\text { R-Cuadrada } \\
\text { Ajd. }\end{array}$ \\
\hline Lineal & 6.78401 & 75.78 & 72.93 \\
Cuadrático & 4.20515 & 92.34 & 89.60 \\
Cúbico Especial & 4.32251 & 92.48 & 89.01 \\
Cúbico & 4.11432 & 94.76 & 90.04 \\
\hline \hline
\end{tabular}

TABLA IX

ANOVA PARA RESISTENCIA A LA COMPRESIÓN

\begin{tabular}{|c|c|c|c|c|c|}
\hline Fuente & $\begin{array}{c}\text { Suma de } \\
\text { Cuadrados }\end{array}$ & Gl & $\begin{array}{c}\text { Cuadrado } \\
\text { Medio }\end{array}$ & $\begin{array}{c}\text { Razón- } \\
\text { F }\end{array}$ & $\begin{array}{l}\text { Valor- } \\
\text { P }\end{array}$ \\
\hline $\begin{array}{l}\text { Modelo } \\
\text { Cuadrático }\end{array}$ & 2961.27 & 4 & 740.318 & 58.81 & 0.0001 \\
\hline Falta de ajuste & 193.668 & 9 & 21.5187 & 1.71 & 0.2646 \\
\hline Error puro & 75.5327 & 6 & 12.5888 & - & - \\
\hline Total (corr.) & 3230.47 & 19 & - & - & - \\
\hline \multicolumn{6}{|c|}{$\begin{array}{l}\text { R-cuadrada }=91.6668 \text { porciento } \\
\text { R-cuadrada (ajustada por g.l. })=89.4447 \text { porciento } \\
\text { Error estándar del est. }=3.54807 \\
\text { Error absoluto medio }=2.99145 \\
\text { Estadístico Durbin-Watson }=2.76322(\mathrm{P}=0.9561) \\
\text { Autocorrelación residual de Lag } 1=-0.399057\end{array}$} \\
\hline Parámetro & Estimado & $\begin{array}{c}\text { Error } \\
\text { Estándar }\end{array}$ & Estadís & tico T & Valor-P \\
\hline $\mathrm{X}_{1}$ : Arcilla & 90.7786 & 5.8413 & - & & - \\
\hline $\mathrm{X}_{2}$ : Arena & 32.4568 & 5.4358 & & & - \\
\hline $\mathrm{X}_{3}$ : Agua & 21.9908 & 12.912 & & & - \\
\hline $\mathrm{X}_{1} \mathrm{X}_{2}$ & -94.5276 & 20.9383 & -4.5145 & & 0.0004 \\
\hline$X_{1} X_{3}$ & -86.8721 & 28.0381 & -3.0983 & & 0.0073 \\
\hline
\end{tabular}

La ecuación del modelo cuadrático ajustado para la resistencia a la compresión está dada por (11). Con respecto a la magnitud de los coeficientes lineales estimados, se puede inferir que la arcilla es el componente en la mezcla que maximiza la resistencia a la compresión del bloque. En cuanto a los efectos combinados se aprecia que tienen un efecto antagónico sobre la respuesta, dado que su signo es negativo.
Cabe resaltar que el valor de los porcentajes de los componentes de la mezcla en el modelo ajustado está expresado en pseudo-componentes.

$$
\begin{aligned}
Y_{1}=90.7786 X_{1} & +32.4568 X_{2}+21.9908 X_{3}-94.5276 X_{1} X_{2} \\
& -86.8721 X_{1} X_{3}
\end{aligned}
$$

La gráfica de la superficie y el contorno de respuesta se muestra en la Fig. 18, en la cual se aprecia que la mayor resistencia a la compresión se logra en mezclas donde el componente arcilla es predominante. La grafica de contornos es de tipo cresta descendente, la cual ocurre cuando se tiene un mínimo, pero el punto estacionario cae fuera de la región experimental [26]. El grafico de trazas en el modelo ajustado para la resistencia a la compresión se ilustra en la Fig. 19. En este se observa que el componente con mayor incidencia en la respuesta es la arcilla; vale la pena resaltar el poco efecto que tiene el agua con respecto a los demás componentes, esto debido a que es el componente con menor magnitud en el coeficiente lineal del modelo ajustado. La gráfica de probabilidad normal de los residuos se muestra en la Fig. 20, la cual proporciona información adicional sobre la calidad del modelo de regresión ajustado. En esta se aprecia que el supuesto de normalidad sobre los errores se cumple razonablemente bien, ya que los puntos en esta gráfica tienden a ajustarse a la línea recta [25]. El gráfico de residuos vs predichos se muestra en la Fig. 21, en esta se observa que los puntos no siguen ningún patrón definido, esto nos indica que el modelo se ajusta de igual manera a lo largo de los valores de Y, y verifica que el modelo es adecuado para explicar la respuesta [25].
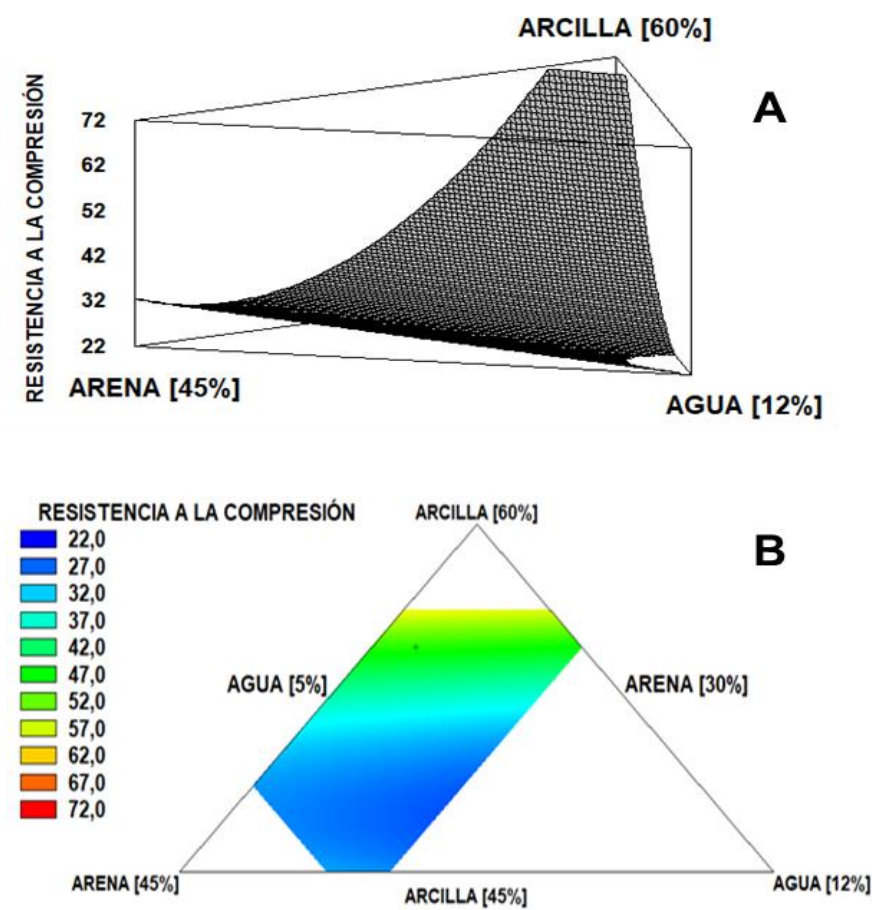

Fig. 18. Gráfica de superficie (A) y contorno (B) de respuesta para la resistencia a la compresión. 


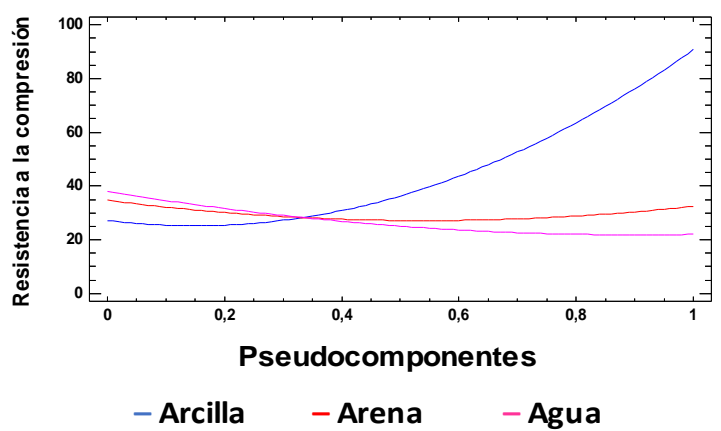

Fig. 19. Gráfica de trazas para la resistencia a la compresión.

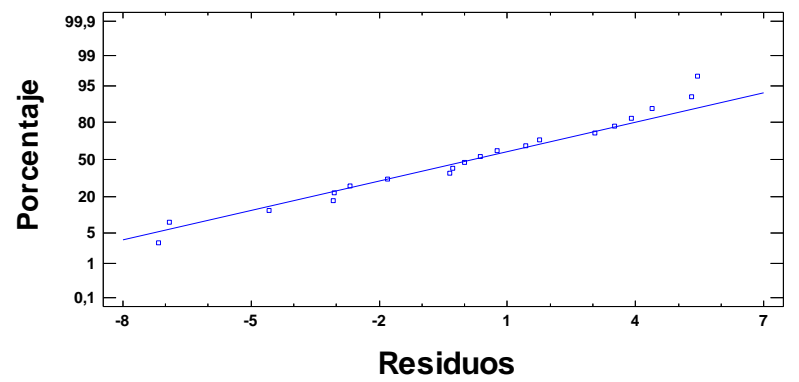

Fig. 20. Gráfica de probabilidad normal de residuos para la resistencia a la compresión.

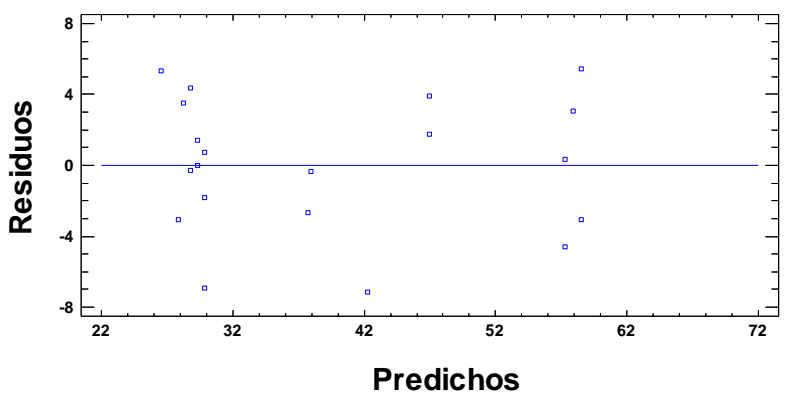

Fig. 21. Gráfica de residuos vs predichos para la resistencia a la compresión.

\section{2) Absorción de agua fría}

Para el porcentaje de absorción de agua fría se obtuvo la Tabla $\mathrm{X}$ donde se muestra el análisis entre los modelos de regresión. En esta se aprecia el valor-P, el cual prueba si ese modelo es estadísticamente significativo cuando se le compara con el cuadrado medio del término de abajo. Normalmente, se seleccionaría un modelo con un valor-P menor que 0.05 , asumiendo que se trabaja al nivel de confianza del 95\% [23]. Teniendo en cuenta este criterio se escogería al modelo lineal.

TABLAX

ANÁLISIS ENTRE LOS MODELOS ESTADÍSTICOS PARA LA ABSORCIÓN DE AGUA FRÍA

\begin{tabular}{llllll}
\hline \hline Fuente & $\begin{array}{c}\text { Suma de } \\
\text { Cuadrados }\end{array}$ & Gl & $\begin{array}{c}\text { Cuadrado } \\
\text { Medio }\end{array}$ & Razón-F & Valor-P \\
\hline Media & 6278.55 & 1 & 6278.55 & & \\
Lineal & 157.342 & 2 & 78.6712 & 24.60 & 0.0000 \\
Cuadrático & 7.64088 & 3 & 2.54696 & 0.76 & 0.5333 \\
Cúbico & 12.1889 & 1 & 12.1889 & 4.59 & 0.0517 \\
Especial & & & & & \\
Cúbico & 6.83678 & 3 & 2.27893 & 0.82 & 0.5105 \\
Error & 27.6911 & 10 & 2.76911 & & \\
Total & 6490.25 & 20 & & & \\
\hline \hline
\end{tabular}

Para la selección del modelo de regresión, además del valor-P, se tiene el criterio de los coeficientes de determinación (R-Cuadrada y R-Cuadrada ajd.) mostrados en la Tabla XI, los cuales muestran el porcentaje de la variación de la respuesta que es explicada por el correspondiente modelo [24]. Para este criterio el modelo lineal también es el indicado, ya que obtiene una R-Cuadrada ajd de al menos $70 \%$. En la Tabla XII se muestra el análisis de varianza para el modelo lineal completo, junto con la estimación de sus coeficientes.

TABLA XI

ANÁLISIS DE COEFICIENTES DE DETERMINACIÓN PARA LA ABSORCIÓN DE AGUA FRÍA

\begin{tabular}{lccc}
\hline \hline \multicolumn{1}{c}{ Modelo } & ES & R-Cuadrada & $\begin{array}{c}\text { R-Cuadrada } \\
\text { Ajd. }\end{array}$ \\
\hline Lineal & 1.78816 & 74.32 & 71.30 \\
Cuadrático & 1.82672 & 77.93 & 70.05 \\
Cúbico & 1.62972 & 83.69 & 76.16 \\
Especial & & & 75.15 \\
Cúbico & 1.66407 & 86.92 & \\
\hline \hline
\end{tabular}

TABLA XII

ANOVA PARA ABSORCIÓN DE AGUA FRÍA

\begin{tabular}{|c|c|c|c|c|c|c|}
\hline Fuente & \multicolumn{2}{|c|}{$\begin{array}{c}\text { Suma de } \\
\text { Cuadrados }\end{array}$} & $\overline{\mathrm{Gl}}$ & $\begin{array}{c}\text { Cuadrado } \\
\text { Medio }\end{array}$ & $\begin{array}{c}\text { Razón- } \\
\text { F }\end{array}$ & $\begin{array}{c}\text { Valor- } \\
\mathrm{P}\end{array}$ \\
\hline Modelo Lineal & \multicolumn{2}{|l|}{157.342} & 2 & 78.6712 & 23.68 & 0.0014 \\
\hline Falta de ajuste & \multicolumn{2}{|l|}{34.4247} & 11 & 3.12951 & 0.94 & 0.5606 \\
\hline Error puro & \multicolumn{2}{|l|}{19.9331} & 6 & 3.32218 & - & - \\
\hline Total (corr.) & \multicolumn{2}{|l|}{211.7} & 19 & 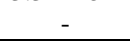 & 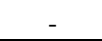 & - \\
\hline $\begin{array}{l}\text { R-cuadrada = } \\
\text { R-cuadrada (a } \\
\text { Error estándar } \\
\text { Error absoluto } \\
\text { Estadístico D } \\
\text { Autocorrelaci } \\
\end{array}$ & $\begin{array}{l}74.3232 \% \\
\text { ajustada por g } \\
\mathrm{r} \text { del est. }=1 . \\
\text { o medio }=1.3 \\
\text { urbin-Watson } \\
\text { ión residual d }\end{array}$ & $\begin{array}{l}.1 .)= \\
8226 \\
36869 \\
=1 \\
\text { e La }\end{array}$ & 71.30 & $\begin{array}{l}\mathrm{P}=0.1588) \\
35906\end{array}$ & & \\
\hline Parámetro & Estimado & & r Esta & dar & lístico T & $\begin{array}{c}\text { Valor- } \\
\mathrm{P}\end{array}$ \\
\hline $\mathrm{X}_{1}$ : Arcilla & 12.1285 & 0.99 & 187 & & - & - \\
\hline $\mathrm{X}_{2}$ : Arena & 22.2231 & 0.99 & 187 & & - & - \\
\hline $\mathrm{X}_{3}$ : Agua & 20.0677 & 2.44 & & & - & - \\
\hline
\end{tabular}

La ecuación del modelo lineal ajustado para la absorción de agua fría está dada por (12). Con respecto a la magnitud de los coeficientes lineales estimados, se puede inferir que la arena es el componente en la mezcla que maximiza el porcentaje de absorción de agua fría del bloque. Cabe resaltar que el valor de los porcentajes de los componentes de la mezcla del modelo ajustado está expresado en pseudo-componentes.

$$
\mathrm{Y}_{2}=12.1285 \mathrm{X}_{1}+22.2231 \mathrm{X}_{2}+20.0677 \mathrm{X}_{3}
$$

La gráfica de la superficie y el contorno de respuesta se muestra en la Fig. 22, en la cual, se aprecia que el mayor porcentaje de absorción de agua fría se logra en mezclas donde el componente arena es predominante. La grafica de contornos es de tipo cordillera estacionaria, la cual es un caso limite, ya sea del máximo o mínimo o del punto de silla, y ocurre cuando uno de los valores propios se aproxima a cero [26]. El gráfico de trazas en el modelo ajustado para el porcentaje de absorción de agua fría se ilustra en la Fig. 23. En este se observa que el componente con mayor incidencia 
en la respuesta es la arena; vale la pena resaltar el poco efecto que tiene la arcilla con respecto a los demás componentes, esto debido a que es el componente con menor magnitud en el coeficiente lineal del modelo ajustado. La gráfica de probabilidad normal de los residuos se muestra en la Fig. 24, la cual proporciona información adicional sobre la calidad del modelo de regresión ajustado. En esta se aprecia que el supuesto de normalidad sobre los errores se cumple razonablemente bien, ya que los puntos en esta gráfica tienden a ajustarse a la línea recta [25]. El gráfico de residuos vs predichos se muestra en la Fig. 25, en este se observa que los puntos no siguen ningún patrón definido, esto nos indica que el modelo se ajusta de igual manera a lo largo de los valores de $\mathrm{Y}$, y verifica que el modelo es adecuado para explicar la respuesta [25].
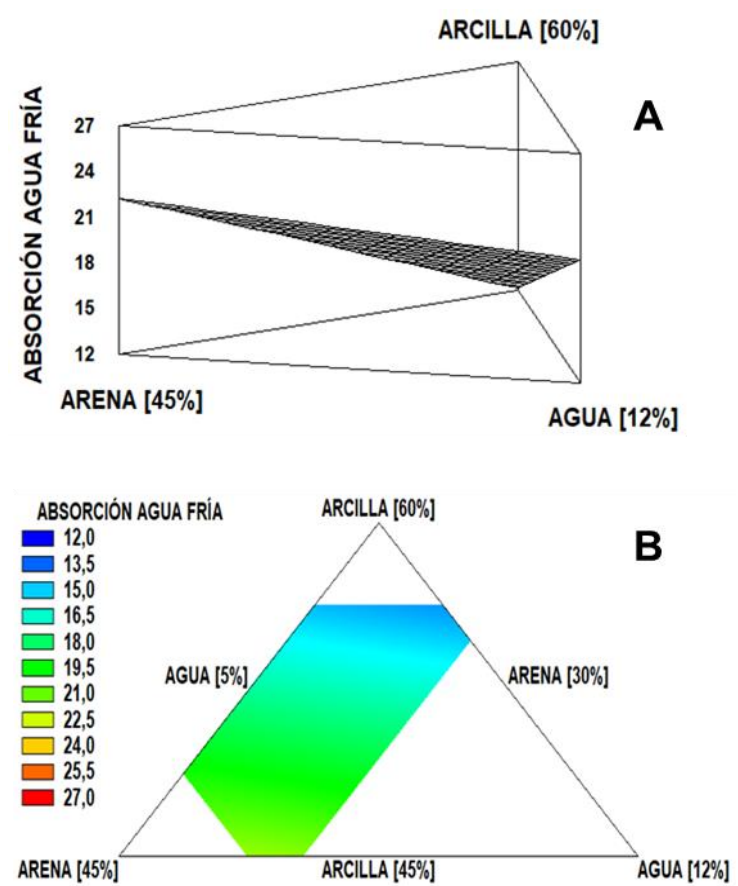

Fig. 22. Gráfica de superficie (A) y contorno (B) de respuesta para la absorción de agua fría.

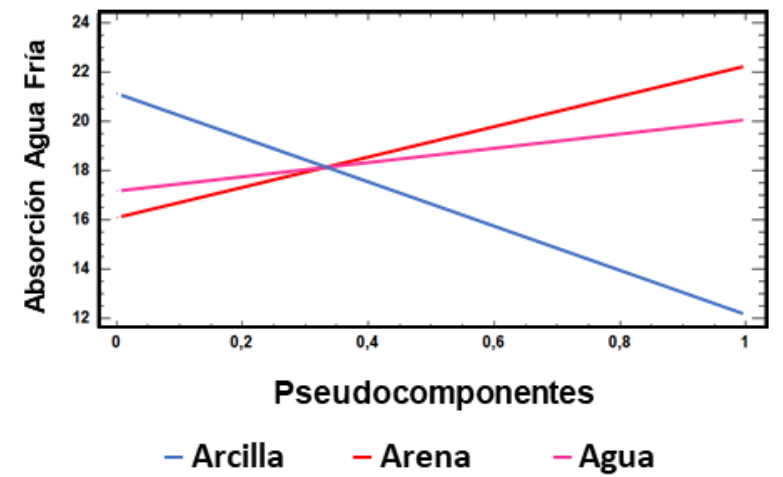

Fig. 23. Gráfica de trazas para el porcentaje de absorción de agua fría.

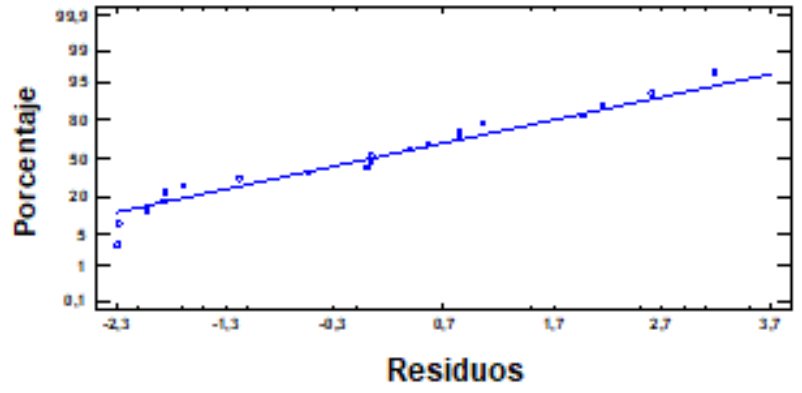

Fig. 24. Gráfica de probabilidad normal de residuos para la absorción de agua fría.

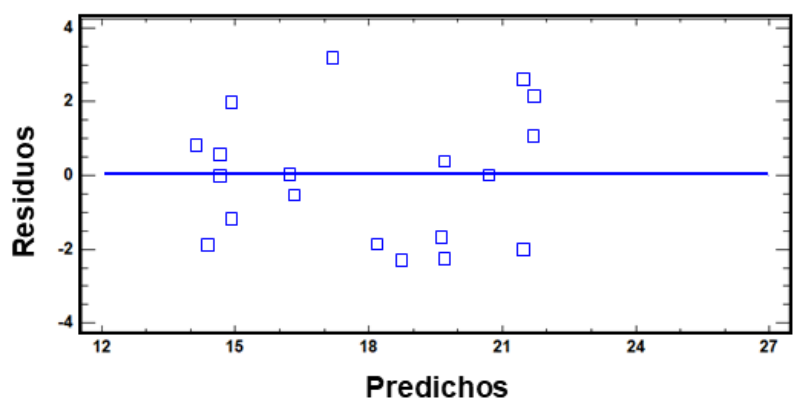

Fig. 25. Gráfica de residuos vs predichos para la absorción de agua fría.

\section{3) Absorción de agua por ebullición}

Para el porcentaje de absorción de agua por ebullición se obtuvo la Tabla XIII donde se muestra el análisis entre los modelos de regresión. En esta se aprecia el valor-P, el cual prueba si ese modelo es estadísticamente significativo cuando se compara con el cuadrado medio del término de abajo. Normalmente, se seleccionaría un modelo con un valor-P menor que 0.05 , asumiendo que se trabaja al nivel de confianza del 95\% [23]. Teniendo en cuenta este criterio se escogería al modelo lineal, pero el valor-P también es menor que 0.05 en el modelo cuadrático, por lo tanto, también podría tenerse en cuenta.

TABLA XIII

ANÁLISIS ENTRE LOS MODELOS ESTADÍSTICOS PARA LA ABSORCIÓN DE AGUA POR EBULLICIÓN

\begin{tabular}{llllll}
\hline \hline Fuente & $\begin{array}{c}\text { Suma de } \\
\text { Cuadrados }\end{array}$ & Gl & $\begin{array}{c}\text { Cuadrado } \\
\text { Medio }\end{array}$ & Razón-F & Valor-P \\
\hline Media & 8770.09 & 1 & 8770.09 & & \\
Lineal & 156.393 & 2 & 78.1963 & 17.13 & 0.0001 \\
Cuadrático & 39.0376 & 3 & 13.0125 & 4.72 & 0.0177 \\
Cúbico & 1.03586 & 1 & 1.03586 & 0.36 & 0.5594 \\
Especial & & & & & \\
Cúbico & 3.22171 & 3 & 1.0739 & 0.31 & 0.8157 \\
Error & 34.303 & 10 & 3.4303 & & \\
Total & 9004.08 & 20 & & & \\
\hline \hline
\end{tabular}

Para la selección del modelo de regresión, además del valor-P, se tiene el criterio de los coeficientes de determinación (R-Cuadrada y R-Cuadrada ajd.) mostrados en la Tabla XIV, los cuales muestran el porcentaje de la variación de la respuesta que es explicada por el correspondiente modelo [24]. Para este criterio el modelo cuadrático es el indicado, ya que maximiza la R-Cuadrada ajd. En la tabla XV se muestra el análisis de varianza para el modelo cuadrático completo, junto 
con la estimación de sus coeficientes.

TABLA XIV

ANÁlISIS DE COEFICIENTES DE DETERMINACIÓN PARA LA ABSORCIÓN DE AGUA POR EBULLICIÓN

\begin{tabular}{lccc}
\hline \hline \multicolumn{1}{c}{ Modelo } & ES & R-Cuadrada & $\begin{array}{c}\text { R-Cuadrada } \\
\text { Ajd. }\end{array}$ \\
\hline Lineal & 2.13649 & 66.84 & 62.94 \\
Cuadrático & 1.65962 & 83.52 & 77.63 \\
Cúbico & 1.69898 & 83.96 & 76.56 \\
Especial & & & 72.15 \\
Cúbico & 1.85211 & 85.34 & 7 \\
\hline \hline
\end{tabular}

TABLA XV

ANOVA PARA ABSORCIÓN DE AGUA POR EBULLICIÓN

\begin{tabular}{|c|c|c|c|c|c|}
\hline Fuente & $\begin{array}{c}\text { Suma de } \\
\text { Cuadrados }\end{array}$ & 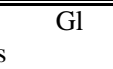 & $\begin{array}{c}\text { Cuadrado } \\
\text { Medio }\end{array}$ & $\begin{array}{c}\text { Razón- } \\
\text { F }\end{array}$ & $\begin{array}{c}\text { Valor- } \\
\text { P }\end{array}$ \\
\hline $\begin{array}{l}\text { Modelo } \\
\text { Cuadrático }\end{array}$ & 2961.27 & 4 & 740.318 & 58.81 & 0.0001 \\
\hline Falta de ajuste & 193.668 & 9 & 21.5187 & 1.71 & 0.2646 \\
\hline Error puro & 75.5327 & 6 & 12.5888 & - & - \\
\hline Total (corr.) & 3230.47 & 19 & - & - & - \\
\hline \multicolumn{6}{|c|}{$\begin{array}{l}\text { R-cuadrada }=77.1143 \text { porciento } \\
\text { R-cuadrada (ajustada por g.1. })=72.8233 \text { porciento } \\
\text { Error estándar del est. }=1.82945 \\
\text { Error absoluto medio }=1.20055 \\
\text { Estadístico Durbin-Watson }=1.95136(\mathrm{P}=0.4583) \\
\text { Autocorrelación residual de Lag } 1=-0.0144869\end{array}$} \\
\hline Parámetro & Estimado & $\begin{array}{c}\text { Error } \\
\text { Estánda }\end{array}$ & Estadi & stico T & Valor-P \\
\hline $\mathrm{X}_{1}$ : Arcilla & 21.1528 & 2.04069 & & & - \\
\hline $\mathrm{X}_{2}$ : Arena & 31.2261 & 2.04069 & & & 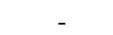 \\
\hline $\mathrm{X}_{3}$ : Agua & 10.3092 & 4.01191 & & - & - \\
\hline $\mathrm{X}_{1} \mathrm{X}_{2}$ & -24.2043 & 9.02974 & -2.680 & & 0.0164 \\
\hline
\end{tabular}

La ecuación del modelo cuadrático ajustado para la absorción de agua por ebullición está dada por (13). Con respecto a la magnitud de los coeficientes lineales estimados, se puede inferir que la arena es el componente en la mezcla que maximiza el porcentaje de absorción de agua por ebullición. En cuanto a los efectos combinados se aprecia que tienen un efecto antagónico sobre la respuesta, dado que su signo es negativo. Cabe resaltar que el valor de los porcentajes de los componentes de la mezcla del modelo ajustado está expresado en pseudo-componentes.

$\mathrm{Y}_{3}=21.1528 \mathrm{X}_{1}+31.2261 \mathrm{X}_{2}+10.3092 \mathrm{X}_{3}-24.2043 \mathrm{X}_{1} \mathrm{X}_{2}$

La gráfica de la superficie y el contorno de respuesta se muestra en la Fig. 26, en la cual, se aprecia que el mayor porcentaje de absorción de agua fría se logra en mezclas donde el componente arena es predominante. La grafica de contornos es de tipo cresta descendente, la cual ocurre cuando se tiene un mínimo, pero el punto estacionario cae fuera de la región experimental [26]. El gráfico de trazas en el modelo ajustado para el porcentaje de absorción de agua por ebullición se ilustra en la Fig. 27. En este se observa que el componente con mayor incidencia en la respuesta es la arena; vale la pena resaltar el poco efecto que tiene el agua con respecto a los demás componentes, esto debido a que es el componente con menor magnitud en el coeficiente lineal del modelo ajustado. La gráfica de probabilidad normal de los residuos se observa en la Fig. 28, la cual proporciona información adicional sobre la calidad del modelo de regresión ajustado. En esta se aprecia que el supuesto de normalidad sobre los errores se cumple razonablemente bien, ya que los puntos en esta gráfica tienden a ajustarse a la línea recta [25]. El gráfico de residuos vs predichos se muestra en la Fig. 29, en la cual, se observa que los puntos no siguen ningún patrón definido, esto nos indica que el modelo se ajusta de igual manera a lo largo de los valores de $\mathrm{Y}$, y verifica que el modelo es adecuado para explicar la respuesta [25].
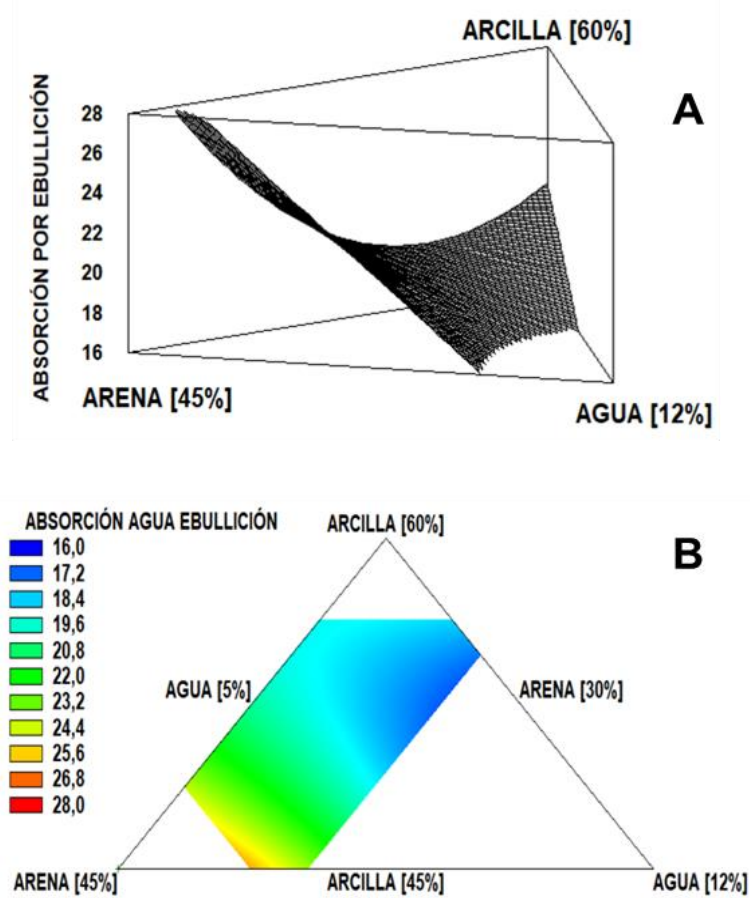

Fig. 26. Gráfica de superficie (A) y contorno (B) de respuesta para la absorción de agua por ebullición.

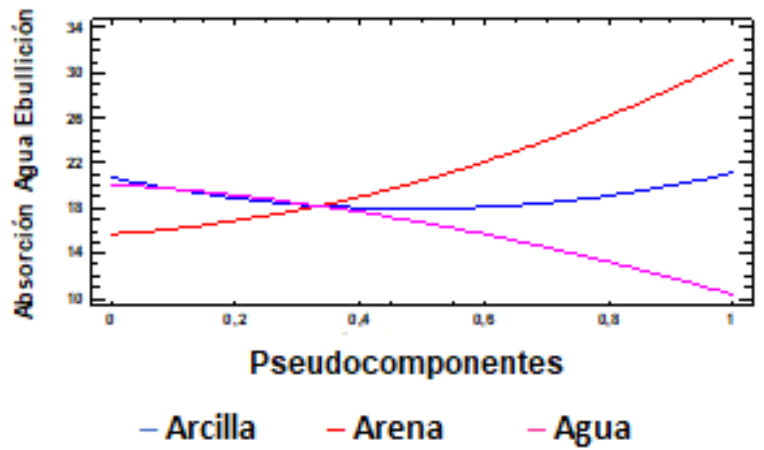

Fig. 27. Gráfica de trazas para el porcentaje de absorción de agua por ebullición. 


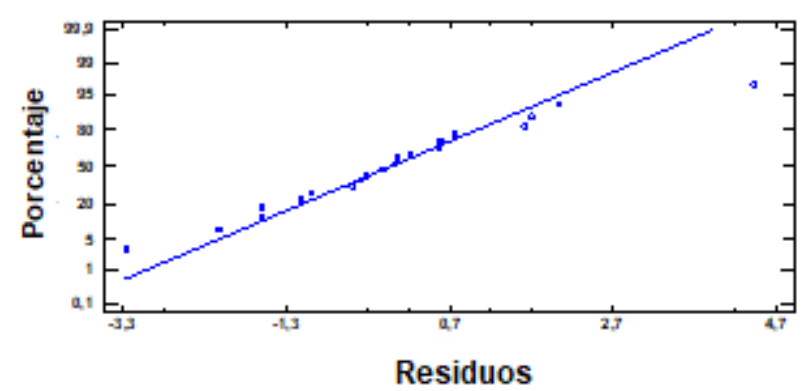

Fig. 28. Gráfica de probabilidad normal de residuos para la absorción de agua por ebullición.

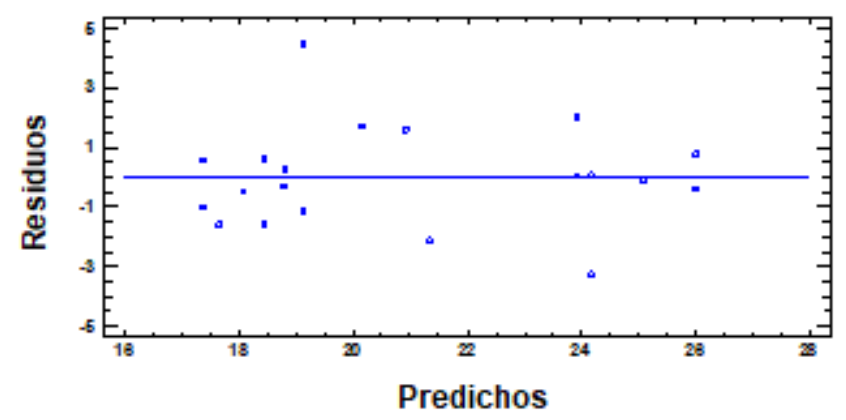

Fig. 29. Gráfica de residuos vs predichos para la absorción de agua por ebullición.

\section{Selección de la Mezcla Óptima para el Bloque}

Para la selección de la mezcla óptima se tuvo en cuenta el criterio de la norma NTC 4205-1 (unidades de mampostería de arcilla cocida. Ladrillos y bloques cerámicos. Parte 1: mampostería estructural), en cuanto a los valores mínimos que deben cumplir los bloques en cada una de las variables de respuesta establecidas. Para la resistencia a la compresión las unidades de perforación horizontal, deben tener un valor mínimo de $35 \mathrm{kgf} / \mathrm{cm}^{2}$ por unidad ensayada; el porcentaje de absorción de agua debe ser máximo de $16 \%$ en unidades saturadas durante $24 \mathrm{~h}$ en agua fría y máximo de $19 \%$ en unidades sumergidas durante $5 \mathrm{~h}$ en agua en ebullición [27].

Para encontrar una mezcla donde todas las respuestas tengan un valor satisfactorio se realizó la optimización simultánea de las variables de respuesta, utilizando la función de deseabilidad de Statgraphics. Este método de optimización consiste en definir una función que estima la deseabilidad global (DG) del producto en cada punto; basta maximizar DG para obtener el punto óptimo buscado [28]. En la Tabla XVI se muestra la función de deseabilidad evaluada en cada punto del diseño. En base a la optimización de la función de deseabilidad, el software calculó las proporciones de los componentes de la mezcla donde todas las respuestas tengan un valor satisfactorio, los resultados se muestran en la Tabla XVII.
TABLA XVI

FUNCIÓN DE DESEABILIDAD PARA CADA PUNTO DEL DISEÑO

\begin{tabular}{lll}
\hline \hline Fila & $\begin{array}{c}\text { Deseabilidad } \\
\text { Prevista }\end{array}$ & $\begin{array}{c}\text { Deseabilidad } \\
\text { Observada }\end{array}$ \\
\hline 1 & 0.484264 & 0.492533 \\
2 & 0.439964 & 0.343963 \\
3 & 0.45716 & 0.737528 \\
4 & 0.255271 & 0.0 \\
5 & 0.563121 & 0.634451 \\
6 & 0.386105 & 0.0 \\
$7\left(^{*}\right)$ & 0.881354 & 0.986488 \\
8 & 0.340844 & 0.411563 \\
9 & 0.589647 & 0.424914 \\
10 & 0.484264 & 0.0 \\
11 & 0.439964 & 0.64703 \\
12 & 0.45716 & 0.439792 \\
13 & 0.255271 & 0.120899 \\
14 & 0.563121 & 0.268732 \\
15 & 0.386105 & 0.371727 \\
16 & 0.643215 & 0.837452 \\
17 & 0.777117 & 0.0 \\
18 & 0.498862 & 0.674168 \\
19 & 0.469974 & 0.0 \\
20 & 0.867898 & 0.642299 \\
\hline \hline${ }^{*}$ Máxima deseabilidad observada.
\end{tabular}

TABLA XVII

PROPORCIÓN ÓPTIMA DE LOS COMPONENTES DE LA MEZCLA

\begin{tabular}{llll}
\hline \hline \multicolumn{1}{c}{ Factor } & Mínimo & Máximo & Óptimo \\
\hline Arcilla & 450 & 600 & 543.091 \\
Arena & 300 & 450 & 375.507 \\
Agua & 50 & 120 & 81.4017 \\
\hline \hline
\end{tabular}

La gráfica de la superficie y el contorno de la función de deseabilidad se muestra en la Fig. 30, en la cual, se aprecia un máximo en 0.986488 significando que la mezcla óptima predicha alcanza un valor deseable cercano al $100 \%$.

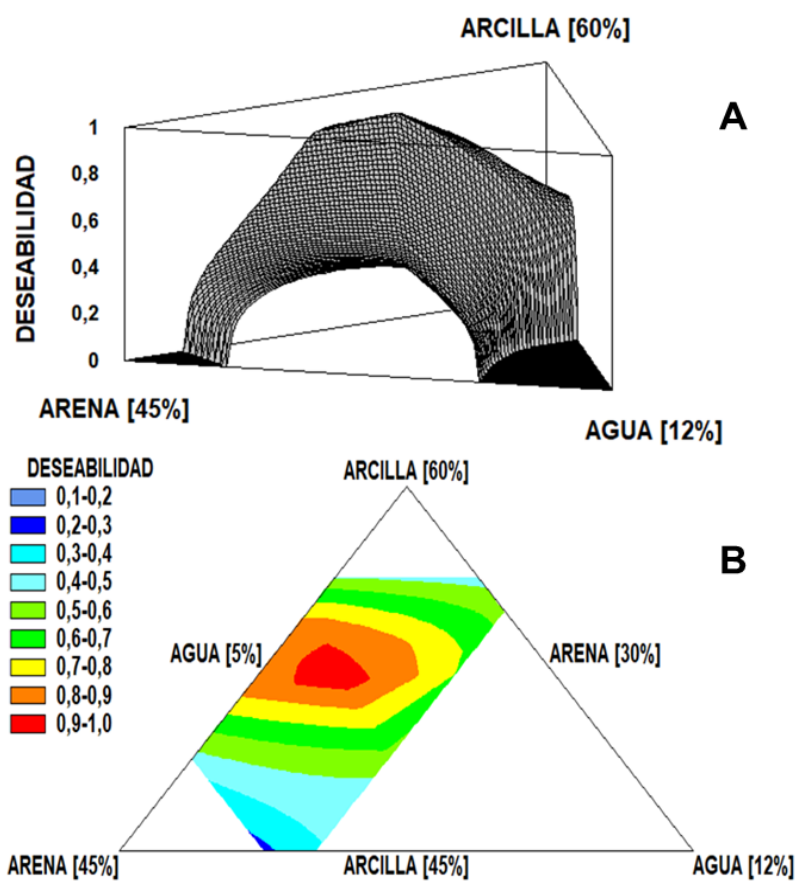

Fig. 30. Gráfica de superficie (A) y contorno (B) de la función deseabilidad. 


\section{INTERPRETACIÓN DE LOS RESULTADOS}

La proporción óptima de los componentes de la mezcla para el bloque samo \#4 se presentó en la Tabla XVII, esta fue establecida teniendo en cuenta los valores observados en el estándar NTC 4205-1 para las variables de respuesta. Sin embargo, es importante validar los resultados obtenidos a través de pruebas de campo. Además, en estas pruebas se verificará si la mezcla seleccionada cumple con otros requerimientos necesarios para obtener un bloque de calidad; tales como color, acabado superficial y dimensiones.

\section{A. Pruebas de Campo}

Se realizaron pruebas de campo a la mezcla óptima seleccionada, siguiendo las consideraciones de las pruebas previas para las variables del proceso. Fueron ensayados cinco lotes de mezcla óptima; cada lote equivalente a 166 bloques en promedio. Con el acompañamiento del departamento de producción de Gres Caribe S.A., se realizaron inspecciones durante el proceso de fabricación para establecer el comportamiento de la nueva mezcla. A continuación, se muestra el cálculo del tiempo de apertura de las válvulas para el suministro de agua en la amasadora y en la bañera de la extrusora para la mezcla establecida. En primera instancia se determina el volumen de agua solicitado para la mezcla, Vs, con (4). De la Tabla XVII, se obtiene la masa de agua solicitada, m. La densidad del agua, $\mathrm{d}_{\mathrm{H} 2 \mathrm{O}}$, es $1000 \mathrm{~kg} / \mathrm{m}^{3}$. Posteriormente se calcula el tiempo de apertura de la electroválvula en la amasadora, $\mathrm{t}_{3}$, usando (5). Similarmente se calcula el tiempo de apertura de la electroválvula en la bañera de la extrusora, $t_{4}$, usando (6). Con los datos arriba presentados se tiene $\mathrm{Vs}=81.4017 \mathrm{lt}, \mathrm{t}_{3}=174.43 \mathrm{seg}, \mathrm{t}_{4}=$ $261.65 \mathrm{seg}$.

\section{1) Pruebas cuantitativas}

Para las pruebas cuantitativas se extrajeron 15 bloques de cada lote, distribuidos para ensayos de esta manera: cinco para el ensayo de resistencia a la compresión, cinco para absorción de agua fría y cinco para absorción de agua por ebullición. Los resultados de cada ensayo se resumen en la Tabla XVIII. Las Fig. 35,36 y 37 presentan los promedios de las corridas para las propiedades compresión, absorción agua fría y por ebullición, respectivamente. Se aprecia que la resistencia a la compresión siempre está, en promedio, por encima del valor exigido por la norma $\left(35 \mathrm{kgf} / \mathrm{cm}^{2}\right)$. Los porcentajes de absorción de agua fría y por ebullición, en promedio, se mantuvieron favorablemente por debajo de los valores máximos permitidos, $16 \%$ y $19 \%$ respectivamente. Únicamente en la corrida 2 se sobrepasó el nivel aceptado, infiriendo a partir de este resultado que no se controlaron correctamente todas las variables fijas, generando un bloque sin las características deseadas.
TABLA XVIII

Resultados Pruebas CuANTitativas a Mezcla Óptima

\begin{tabular}{|c|c|c|c|}
\hline Lote & $\begin{array}{c}\text { Res. Comp. } \\
{\left[\mathrm{kgf} / \mathrm{cm}^{2}\right]}\end{array}$ & $\begin{array}{l}\text { Abs. Agua } \\
\text { Fría [\%] }\end{array}$ & $\begin{array}{c}\text { Abs. por } \\
\text { Ebullición [\%] }\end{array}$ \\
\hline $\begin{array}{l}\text { Lote 1: } \\
\text { (167 } \\
\text { Bloques) }\end{array}$ & $\begin{aligned} \mathrm{M}_{1} & =40.21 \\
\mathrm{M}_{2} & =52.56 \\
\mathrm{M}_{3} & =31.11 \\
\mathrm{M}_{4} & =37.82 \\
\mathrm{M}_{5} & =43.03 \\
\text { Prom } & =40.95\end{aligned}$ & $\begin{array}{c}\mathrm{M}_{6}=17.74 \\
\mathrm{M}_{7}=12.02 \\
\mathrm{M}_{8}=14.23 \\
\mathrm{M}_{9}=16.68 \\
\mathrm{M}_{10}=16.44 \\
\text { Prom }=15.42\end{array}$ & $\begin{aligned} \mathrm{M}_{11} & =15.21 \\
\mathrm{M}_{12} & =20.59 \\
\mathrm{M}_{13} & =18.42 \\
\mathrm{M}_{14} & =21.29 \\
\mathrm{M}_{15} & =18.77 \\
\text { Prom } & =18.85\end{aligned}$ \\
\hline $\begin{array}{l}\text { Lote 2: } \\
\text { (170 } \\
\text { Bloques) }\end{array}$ & $\begin{aligned} M_{1} & =36.75 \\
M_{2} & =42.24 \\
M_{3} & =44.96 \\
M_{4} & =28.05 \\
M_{5} & =34.16 \\
\text { Prom } & =37.23\end{aligned}$ & $\begin{array}{c}\mathrm{M}_{6}=18.65 \\
\mathrm{M}_{7}=13.39 \\
\mathrm{M}_{8}=19.44 \\
\mathrm{M}_{9}=15.96 \\
\mathrm{M}_{10}=17.93 \\
\text { Prom }=17.07\end{array}$ & $\begin{aligned} \mathrm{M}_{11} & =21.04 \\
\mathrm{M}_{12} & =15.32 \\
\mathrm{M}_{13} & =22.74 \\
\mathrm{M}_{14} & =20.51 \\
\mathrm{M}_{15} & =16.68 \\
\text { Prom } & =19.26\end{aligned}$ \\
\hline $\begin{array}{l}\text { Lote 3: } \\
\text { (162 } \\
\text { Bloques) }\end{array}$ & $\begin{aligned} \mathrm{M}_{1} & =52.36 \\
\mathrm{M}_{2} & =38.23 \\
\mathrm{M}_{3} & =41.63 \\
\mathrm{M}_{4} & =44.84 \\
\mathrm{M}_{5} & =39.09 \\
\text { Prom } & =43.23\end{aligned}$ & $\begin{array}{c}\mathrm{M}_{6}=16.24 \\
\mathrm{M}_{7}=13.55 \\
\mathrm{M}_{8}=14.75 \\
\mathrm{M}_{9}=17.91 \\
\mathrm{M}_{10}=16.51 \\
\text { Prom }=15.79\end{array}$ & $\begin{aligned} M_{11} & =13.54 \\
M_{12} & =20.58 \\
M_{13} & =16.93 \\
M_{14} & =15.42 \\
M_{15} & =19.23 \\
\text { Prom } & =17.14\end{aligned}$ \\
\hline $\begin{array}{l}\text { Lote 4: } \\
\text { (166 } \\
\text { Bloques) }\end{array}$ & $\begin{aligned} M_{1} & =35.21 \\
M_{2} & =25.87 \\
M_{3} & =49.36 \\
M_{4} & =37.55 \\
M_{5} & =32.27 \\
\text { Prom } & =36.05\end{aligned}$ & $\begin{array}{c}\mathrm{M}_{6}=10.22 \\
\mathrm{M}_{7}=16.33 \\
\mathrm{M}_{8}=19.52 \\
\mathrm{M}_{9}=16.61 \\
\mathrm{M}_{10}=15.48 \\
\text { Prom }=15.63\end{array}$ & $\begin{aligned} M_{11} & =19.57 \\
M_{12} & =18.33 \\
M_{13} & =22.03 \\
M_{14} & =16.32 \\
M_{15} & =15.95 \\
\text { Prom } & =18.44\end{aligned}$ \\
\hline $\begin{array}{l}\text { Lote 5: } \\
\text { (165 } \\
\text { Bloques) }\end{array}$ & $\begin{aligned} \mathrm{M}_{1} & =53.2 \\
\mathrm{M}_{2} & =43.75 \\
\mathrm{M}_{3} & =39.68 \\
\mathrm{M}_{4} & =35.34 \\
\mathrm{M}_{5} & =34.88 \\
\text { Prom } & =41.38\end{aligned}$ & $\begin{aligned} \mathrm{M}_{6} & =10.46 \\
\mathrm{M}_{7} & =15.21 \\
\mathrm{M}_{8} & =16.59 \\
\mathrm{M}_{9} & =11.33 \\
\mathrm{M}_{10} & =16.03 \\
\text { Prom } & =13.92\end{aligned}$ & $\begin{array}{r}\mathrm{M}_{11}=17.77 \\
\mathrm{M}_{12}=23.18 \\
\mathrm{M}_{13}=15.74 \\
\mathrm{M}_{14}=20.02 \\
\mathrm{M}_{15}=12.22 \\
\text { Prom }=17.78\end{array}$ \\
\hline
\end{tabular}

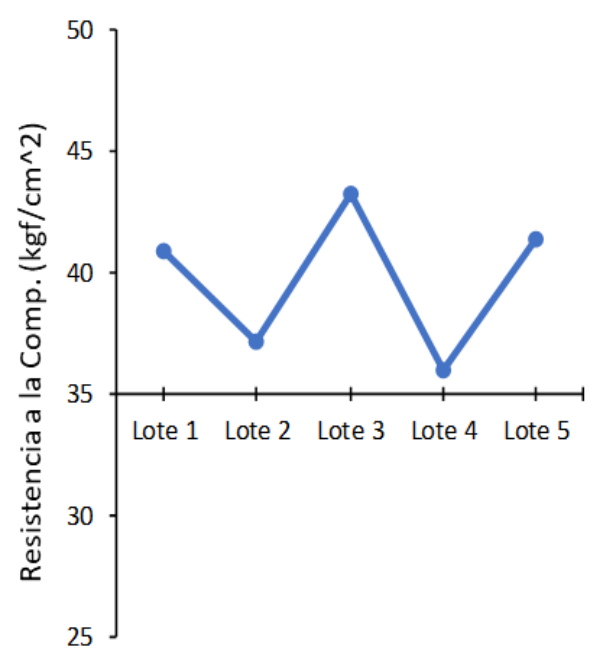

Fig. 35. Resistencia a la compresión promedio de las cinco muestras ensayadas. 


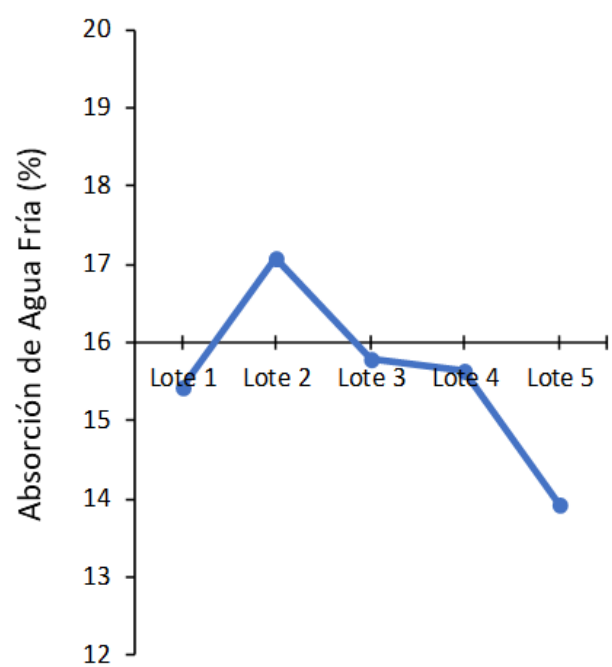

Fig. 36. Porcentaje de absorción de agua fría promedio de las cinco muestras ensayadas.

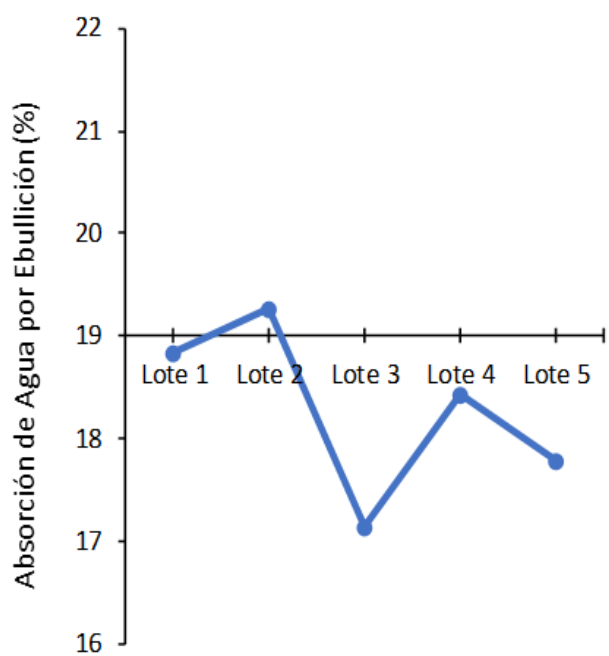

Fig. 37. Porcentaje de absorción de agua por ebullición promedio de las cinco muestras ensayadas.

\section{2) Pruebas cualitativas}

Para determinar la calidad de los bloques, no basta con las pruebas cuantitativas presentadas. Se requiere que el producto cumpla con unos requerimientos cualitativos mínimos para satisfacción del cliente, tales como acabado superficial, color y dimensiones. Se inspeccionó la totalidad de los bloques producidos en cada lote y se cuantificaron los que tuvieran defectos de este tipo, catalogados como bloques de segunda y que son ofrecidos como productos con defecto a menor precio. Tales bloques presentan betas o color opaco evidente, deformaciones excesivas, grietas muy marcadas y dimensiones inaceptables. Las Tablas IXX y XX presentan el conteo de bloques de segunda y de desperdicio. Estos últimos se determinan de acuerdo al aspecto o defecto que impide la comercialización. También se cuentan en esta tabla los bloques que estallan en la cocción. En la Fig. 31 se muestra el gráfico circular con la distribución de calidad en las pruebas realizadas a la mezcla óptima.
TABLA IXX

Resultados Pruebas Cualitativas a Mezcla Óptima

\begin{tabular}{|c|c|c|c|c|c|c|}
\hline Lote & Color & & Acabado Su & Ificial & "Dimensiones & \\
\hline $\begin{array}{c}\text { Lote } \\
1\end{array}$ & $\begin{array}{c}\text { Bloque } \\
\text { con } \\
\text { betas u } \\
\text { opaco }\end{array}$ & $\begin{array}{c}3 \\
1.8 \%\end{array}$ & $\begin{array}{c}\text { Bloques } \\
\text { deformados } \\
\text { Bloques } \\
\text { Agrietados }\end{array}$ & $\begin{array}{c}5 \\
3.0 \% \\
\\
7 \\
4.2 \%\end{array}$ & $\begin{array}{l}\text { Bloques de } \\
\text { dimensión } \\
\text { inaceptable }\end{array}$ & $\begin{array}{l}3 \\
1.8 \%\end{array}$ \\
\hline $\begin{array}{c}\text { Lote } \\
2\end{array}$ & $\begin{array}{c}\text { Bloque } \\
\text { con } \\
\text { betas u } \\
\text { opaco }\end{array}$ & $\begin{array}{c}5 \\
3.0 \%\end{array}$ & $\begin{array}{c}\text { Bloques } \\
\text { deformados }\end{array}$ & $\begin{array}{c}2 \\
1.2 \%\end{array}$ & $\begin{array}{l}\text { Bloques de } \\
\text { dimensión } \\
\text { inaceptable }\end{array}$ & $\begin{array}{l}7 \\
4.1 \%\end{array}$ \\
\hline $\begin{array}{c}\text { Lote } \\
3\end{array}$ & $\begin{array}{c}\text { Bloque } \\
\text { con } \\
\text { betas u } \\
\text { opaco }\end{array}$ & $\begin{array}{c}8 \\
5.0 \%\end{array}$ & $\begin{array}{c}\text { Bloques } \\
\text { deformados } \\
\text { Bloques } \\
\text { Agrietados }\end{array}$ & $\begin{array}{c}3 \\
1.9 \%\end{array}$ & $\begin{array}{l}\text { Bloques de } \\
\text { dimensión } \\
\text { inaceptable }\end{array}$ & $\begin{array}{l}5 \\
3.1 \%\end{array}$ \\
\hline $\begin{array}{c}\text { Lote } \\
4\end{array}$ & $\begin{array}{c}\text { Bloque } \\
\text { con } \\
\text { betas u } \\
\text { opaco }\end{array}$ & $\begin{array}{c}7 \\
4.2 \%\end{array}$ & $\begin{array}{c}\text { Bloques } \\
\text { deformados }\end{array}$ & $\begin{array}{c}3 \\
1.8 \%\end{array}$ & $\begin{array}{l}\text { Bloques de } \\
\text { dimensión } \\
\text { inaceptable }\end{array}$ & $\begin{array}{l}3 \\
1.8 \%\end{array}$ \\
\hline $\begin{array}{c}\text { Lote } \\
5\end{array}$ & $\begin{array}{c}\text { Bloque } \\
\text { con } \\
\text { betas u } \\
\text { opaco }\end{array}$ & $\begin{array}{c}3 \\
1.8 \%\end{array}$ & $\begin{array}{c}\text { Bloques } \\
\text { deformados } \\
\text { Bloques } \\
\text { Agrietados }\end{array}$ & $\begin{array}{c}2 \\
1.2 \%\end{array}$ & $\begin{array}{l}\text { Bloques de } \\
\text { dimensión } \\
\text { inaceptable }\end{array}$ & $\begin{array}{c}2 \\
1.2 \%\end{array}$ \\
\hline
\end{tabular}

TABLA XX

CARACTERÍSTICAS DE CALIDAD DE LA MEZCla ÓPTIMA

\begin{tabular}{llll}
\hline \hline \multicolumn{1}{c}{ Lote } & $\begin{array}{c}\text { Bloques de } \\
\text { Segunda }\end{array}$ & $\begin{array}{c}\text { Bloques de } \\
\text { Desperdicio }\end{array}$ & $\begin{array}{c}\text { Bloques de Baja } \\
\text { Calidad }\end{array}$ \\
\hline $\begin{array}{c}\text { Lote 1: } \\
\text { (167 Bloques) }\end{array}$ & $7(4.2 \%)$ & $5(3.0 \%)$ & $12(7.2 \%)$ \\
$\begin{array}{c}\text { Lote 2: } \\
\text { (170 Bloques) }\end{array}$ & $7(4.1 \%)$ & $8(4.7 \%)$ & $15(8.8 \%)$ \\
$\begin{array}{l}\text { Lote 3: } \\
\text { (162 Bloques) }\end{array}$ & $11(6.8 \%)$ & $5(3.1 \%)$ & $16(9.9 \%)$ \\
$\begin{array}{l}\text { Lote 4: } \\
(166 \text { Bloques) }\end{array}$ & $15(9.0 \%)$ & $7(4,2 \%)$ & $22(13.2 \%)$ \\
$\begin{array}{l}\text { Lote 5: } \\
(165 \text { Bloques })\end{array}$ & $3(1.8 \%)$ & $11(6.7 \%)$ & $14(8.5 \%)$ \\
Promedio & $5.18 \%$ & $4.34 \%$ & $9.52 \%$ \\
\hline \hline
\end{tabular}

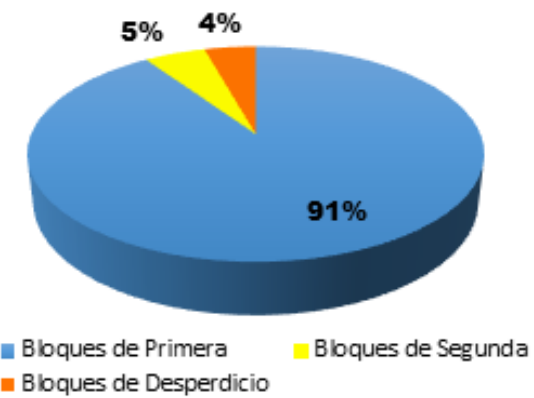

Fig. 31. Gráfico circular distribución de calidad en las pruebas realizadas a la mezcla óptima. 
En las cinco pruebas de campo realizadas, se encontró un promedio de $9.52 \%$ de bloques de baja calidad, que corresponden a la suma del porcentaje de los bloques de segunda y los bloques de desperdicio. Se observa un porcentaje promedio menor del $10 \%$, un resultado favorable teniendo en cuenta que la empresa venia presentando porcentajes mayores de $10 \%$ de bloques de mala calidad para el bloque samo \#4.

\section{B. Análisis de Costos}

Para el análisis de costos en la fabricación del bloque samo \#4, se tuvo como referencia únicamente el valor de las materias primas utilizadas. Los valores fueron suministrados por Gres Caribe S.A. En las Tablas XXI y XXII, se muestra el comparativo de costos entre la fabricación del bloque con la mezcla actual y utilizando la nueva mezcla propuesta. En estas se aprecia que usando la nueva mezcla se logra una disminución de \$0.99 COP por kg de mezcla y de \$9.606 COP por bloque. Esto es debido a la disminución del consumo de arcilla que es el material con mayor costo comercial. En promedio la empresa produce mensualmente 1’327.500 bloques \#4, por lo tanto, si se implementa la nueva mezcla se obtendría un ahorro mensual de \$12’751.965 COP.

TABLA XXI

Costos de Fabricación del BloQue con la Mezcla ACTUAL

\begin{tabular}{|c|c|c|c|}
\hline Materias Primas & $\begin{array}{l}\text { Costo } \\
{[\$ / \mathrm{kg}]}\end{array}$ & $\begin{array}{c}\text { Dosificación } \\
{[\%]}\end{array}$ & $\begin{array}{c}\text { Costo de } \\
\text { Mezcla }[\$ / \mathrm{kg}]\end{array}$ \\
\hline Arcilla & 24.55 & 54.3091 & 24.55 \\
\hline Arena & 8.22 & 37.5507 & 8.22 \\
\hline agua & 0.285 & 8.14017 & 0.285 \\
\hline \multicolumn{3}{|c|}{ Costo Total por $\mathrm{kg}$ de mezcla $[\$ / \mathrm{kg}]$} & 33.05 \\
\hline \multicolumn{3}{|c|}{ Peso del Bloque Samo \#4 Crudo [kg] } & 6.3 \\
\hline \multicolumn{3}{|c|}{ Costo Total por Bloque $[\$]$} & 208.25 \\
\hline
\end{tabular}

TABLA XXII

COSTOS DE FABRICACIÓN DEL BLOQUE CON LA MEZCla ACTUAL

\begin{tabular}{|c|c|c|c|}
\hline Materias Primas & $\begin{array}{l}\text { Costo } \\
{[\$ / \mathrm{kg}]}\end{array}$ & $\begin{array}{c}\text { Dosificación } \\
{[\%]}\end{array}$ & $\begin{array}{c}\text { Costo de } \\
\text { Mezcla }[\$ / \mathrm{kg}]\end{array}$ \\
\hline Arcilla & 45.2 & 60 & 27.12 \\
\hline Arena & 21.9 & 30 & 6.57 \\
\hline agua & 3.5 & 10 & 0.35 \\
\hline \multicolumn{3}{|c|}{ Costo Total por kg de mezcla $[\$ / \mathrm{kg}]$} & 34.04 \\
\hline \multicolumn{3}{|c|}{ Peso del Bloque Samo \#4 Crudo [kg] } & 6.4 \\
\hline \multicolumn{3}{|c|}{ Costo Total por Bloque $[\$]$} & 217.856 \\
\hline
\end{tabular}

\section{CONCLUSIONES}

En este artículo se utilizó la metodología estadística conocida como diseño de experimentos para encontrar la proporción óptima de los componentes de la mezcla para el bloque samo \#4 en la empresa Gres Caribe S.A. Este estudio se realizó considerando los requerimientos exigidos por el estándar NTC 4205-1 para resistencia a la compresión y absorción de agua. El análisis estadístico de los datos recolectados fue asistido con Statgraphics Centurion XVI. Se seleccionaron los puntos experimentales y luego fueron generados los modelos matemáticos que describen el comportamiento de las variables de salida, para obtener la proporción óptima de los componentes de la mezcla para el bloque. Finalmente, se validaron los resultados obtenidos a través de pruebas de campo de la mezcla encontrada.

Las pruebas de campo preliminares permitieron determinar las restricciones a los componentes de la mezcla, los porcentajes iniciales fueron sugeridos de acuerdo a la experiencia e intuición del equipo técnico de operarios e ingenieros que participa en el proceso de fabricación del bloque. Estas pruebas evidenciaron importantes variaciones de propiedades, tanto en la fabricación del bloque como en el producto terminado. Fue encontrado que bloques con porcentaje de arcilla entre $45 \%$ y $60 \%$ presentaron mejores características. Para la arena, el porcentaje adecuado establecido fue entre $30 \%$ y $45 \%$. Similarmente, un rango de $5 \%$ al $12 \%$ de agua, en peso, es adecuado para la fabricación del bloque. A partir de los límites de cada componente, se estableció la región experimental, y se propusieron los puntos candidatos de mezcla con el modelo cúbico completo. Sin embargo, las mezclas candidatas se duplicaron con la finalidad de que el software seleccione, a través del criterio D-óptimo, los veinte puntos del diseño de una lista de cuarenta corridas posibles, y así poder garantizar réplicas que permitan estimar el error experimental y verificar la adecuación del modelo.

A partir de los resultados de los experimentos realizados se ajustó el modelo de regresión y se caracterizó la superficie de respuesta para cada variable establecida. El modelo de regresión para la resistencia a la compresión fue del tipo cuadrático, el cual se ajusta a los datos experimentales en aproximadamente un $89.6 \%$. Para la absorción de agua fría bastó un modelo de regresión lineal, este se ajusta a los datos experimentales en un $71.3 \%$. La absorción de agua por ebullición usó un modelo de regresión cuadrático, con $77.6 \%$ de ajuste a los datos experimentales. Mediante el uso de Statgraphics Centurion XVI se determinó la mezcla óptima para el bloque, a través de la maximización de la función de deseabilidad, esta es $54.3091 \%$ de arcilla, $37.5507 \%$ de arena y $8.14017 \%$ de Agua.

Para validar los resultados obtenidos, se realizaron unas pruebas de campo finales. Estas consistieron en ensayar cinco lotes de la nueva mezcla encontrada para establecer si el producto cumple con unos requerimientos del tipo cuantitativo y cualitativo. Se encontró que con la nueva mezcla se obtienen resultados favorables en cuanto a la resistencia a la compresión, con un valor promedio mínimo de $36.05 \mathrm{kgf} / \mathrm{cm} 2$, superior al mínimo establecido por la norma. En cuanto a la absorción de agua fría y por ebullición, estas se mantuvieron por debajo del límite establecido por norma, 16\% y $19 \%$ respectivamente, excepto en la segunda corrida debido a mal control del experimento. En conjunto con el departamento de producción de la empresa, se pudo interpretar que la proporción de la mezcla encontrada produce en promedio bloques que cumplen con el estándar NTC 4205-1 para una unidad ensayada, en resistencia a la compresión y absorción de agua. Además, se obtuvieron en promedio porcentajes menores del $10 \%$ entre bloques de segunda y bloques de desperdicio. Con esta nueva mezcla propuesta se logra un ahorro de $\$ 9.606$ por cada bloque fabricado, debido a que se 
disminuye el consumo de arcilla que es el material mayor costo comercial. Este ahorro es equivalente a una disminución porcentual de $4.41 \%$, cuantificada en $\$ 153^{\prime} 023.580$ al año, resultado satisfactorio para la empresa.

\section{AGRADECIMIENTOS}

Los autores agradecen el apoyo de la Facultad de Ingeniería y la Vicerrectoría de Investigaciones, Extensión y Proyección Social de la Universidad del Atlántico para la realización de este proyecto.

\section{REFERENCIAS}

[1] P. M. Rainusso, "Metodología para la aplicación del diseño de experimentos (DoE) en la industria," Tesis Doctoral, Univ. de Navarra, San Sebastián, España, 2018.

[2] D. C. Montgomery, "Introducción," en Diseño y análisis de experimentos, 2da ed., México D. F., México: Limusa Wiley, 2004, p. 120. [En línea]. Disponible: https://libreria-limusa.com/producto/diseno$\mathrm{y}$-analisis-de-experimentos-2a-ed/

[3] R. O. Kuehl, "Principios para el diseño de investigaciones," en Diseño de experimentos, 2da ed., México D. F., México: Thomson Learning, 2001, pp. 1-36. [En línea]. Disponible: https://wiartur.files.wordpress.com/2010/04/kuehl-diseno-deexperimentos.pdf

[4] D. C. Pérez, M. O. Bustamante, D. F. Gutiérrez y A. A. Correa, "Diseño de mezclas en formulaciones industriales," Dyna, vol. 82, no. 189, pp. 149-156, Feb. 2015. DOI:10.15446/dyna.v82n189.42785

[5] H. Saltos y A. Bayas, "Aplicación de un diseño experimental de mezclas en el desarrollo de una barra energética con base en el salvado de palmito de pejibaye (Bactris gasipaes H.B.K.)," Revista Tecnológica ESPOL-RTE, vol. 23, no. 2, pp. 1-8, Nov. 2010.

[6] J. Chu, G. Amidon, N. Weiner y A. Goldberg, "Mixture experimental design in the development of a mucoadhesive gel formulation," Pharmaceutical Research, vol. 8, no. 11, pp. 1401-1407, Jun. 1991. DOI:10.1023/A:1015853223929

[7] S. Correia, D. Hotza y A. Segadaes, "Optimización de la resistencia mecánica y densidad de cerámicas en verde a través del diseño de mezclas," Boletín Sociedad. Española de Cerámica y Vidrio, vol. 44, no. 1, pp. 53-58, 2005. DOI: $10.3989 /$ cyv.2005.v44.i1.403

[8] S. F. Guerrero, "Optimización del proceso de mezcla de arcilla para la producción de ladrillos, en el sector artesanal," Trabajo de Grado, Fac. Ciencias Químicas, Univ. de Cuenca, Cuenca, Ecuador, 2014.

[9] H. Pulido y R. Salazar, "Introducción al diseño de experimentos," en Análisis y diseño de experimentos, 2da ed., México D. F., México: MacGraw Hill, 2008, pp. 2-17. [En línea]. Disponible: http://gc.initelabs.com/recursos/files/r161r/w19537w/analisis_y_diseno_ experimentos.pdf

[10] S. Correia, E. Grun, C. Denardi, D. Hotza y M. Folgueras, "Using experiments design to model the effect to raw materials on the sintering and technological properties of bricks compositions," Materials Science Forum, vol. 516, no. 1, pp. 1424-1428, May. 2006. DOI: 10.4028/www.scientific.net/MSF.514-516.1424

[11] D. Onwuca, C. Chijioke y S. Onwuca, "Prediction of the crushing strength of sda-clay fired bricks using simplex theory," IOSR-JMCE, vol. 5, no. 6, pp. 52-58, Abr. 2013. DOI:10.9790/1684-0565258

[12] D. Onwuka, C. chiemela, N. Ibearugbulam y O. Adinna, "Computer program for the determination of optimum compressive strength of sdaclay bricks," International Journal of Scientific and Research Publications, vol. 4, no. 4, pp. 1-3, Mar. 2014.

[13] R. H. Myers, D. C. Montgomery y C. M. Anderson-Cook, "Other mixture design and analysis techniques," in Response surface methodology, 3rd ed., Hoboken, NJ, USA: Wiley, 2009, pp. 589-643.

[14] X. Jin, Y. Zhang, L. Xiao y Z. Zhao, "Optimization of extended zeroorder release gliclazide tablets using D-optimal mixture design," Yakugaku Zasshi, vol. 128, no. 10, pp. 1475-1483, Jun. 2008. DOI: 10.1248/yakushi.128.1475
[15] Boletín de coyuntura económica de Barranquilla, 1er trimestre, Cámara de Comercio, Barranquilla, Colombia, 2016, pp. 14-16. [En línea]. Disponible: http://www.camarabaq.org.co/boletines-de-coyuntura/

[16] E. Besoain, "Introducción," en Mineralogía de arcillas de suelos, vol. 1, San José, Costa Rica: IICA, 1985, pp. 7-39.

[17] M. Vélez, A. Cedeño y C. González, "Materias primas para la industria cerámica: beneficio y procesamiento," LatinAmerican. Journal of Metallurgy and Materials, vol. 10, no. 1-2, pp. 74-77, 1990.

[18] S. M. Rincón, J. Molina y D. C. Rozo, "Propiedades físico mecánicas de bloques H10 fabricados en el área metropolitana de Cúcuta," Ciencia e Ingeniería Neogranadina, vol. 24, no. 1, pp. 67-78, Mar. 2014. DOI: $10.18359 / \mathrm{rcin} .8$

[19] J. D. Santos, P. Y. Malagón y E. M. Córdoba, "Caracterización de arcillas y preparación de pastas cerámicas para la fabricación de tejas y ladrillos en la región de Barichara, Santander," Dyna, vol. 78, no. 167, pp. 50-58, Jun. 2011.

[20] J. F. Gelves, J. Sánchez y G. Peña Rodríguez, “Comportamiento de las arcillas del área metropolitana de Cúcuta sometidas a proceso de moldeo por extrusión," Respuestas, vol. 14, no. 2, pp. 32-38, Nov. 2009.

[21] J. A. Muñoz Chaves, R. A. Muñoz Menese, P. Mancill y J. E. Rodríguez Páez, "Estudio del procesamiento cerámico de las arcillas de la vereda "La Codicia" (Guapi, Colombia) para potenciar su uso en la elaboración de piezas cerámicas," Rev. Fac. Ing. Univ. Antioquia, vol. 1, no. 42, pp. 68-78, Dic. 2007.

[22] R. E. Walpole, R. H. Myers, S. L. Myers y K. Ye, "Experimentos factoriales (dos o más factores)," en Probabilidad y estadística para ingeniería y ciencias, 9na ed., Naucalpan de Juárez, México: Pearson, 2012, pp. 561-596. [En línea]. Disponible: http://www.pearsonenespanol.com/mexico/educacionsuperior/walpole_inde

[23] G. Kaya Uyanik y N. Guler, "A study on multiple linear regression analysis," 4th International Conference on New Horizons in Education, Sakarya, Turkey, 2013, pp. 234-240. DOI: 10.1016/j.sbspro.2013.12.027

[24] J. O. Rawlings, S. G. Pantula y D. A. Dickey, "Analysis of variance and quadratic forms," in Applied Regression Analysis, 2nd ed., New York, NY, USA: Springer, 1998, pp. 101-160. DOI: 10.1007/b98890

[25] D. C. Montgomery, E. A. Peck y G. Geoffrey Vinning, "Model adequacy checking," in Introduction to linear regression analysis, 5th ed., Hoboken, NJ, USA: Wiley, 2012, pp. 129-170. [En línea]. Disponible: $\quad$ https://www.wiley.com/enco/Introduction+to+Linear+Regression+Analysis, +5 th+Edition-p9780470542811

[26] Y. Wang, L. Deng y Y. Fan, "Preparation of soy-based adhesive enhanced by waterborne polyurethane: optimization by response surface methodology," Adv. Mater. Sci. Eng., vol. 2018, pp. 1-8, Jun. 2018. DOI: $10.1155 / 2018 / 9253670$

[27] Unidades de Mampostería de Arcilla Cocida. Ladrillos y Bloques Cerámicos. Parte 1: Mampostería Estructural, NTC 4205-1, ICONTEC, $2009 . \quad$ [En línea]. Disponible: https://tienda.icontec.org/producto/ntc4205-1-2/?v=42983b05e2f2

[28] M. Núñez, "Optimización de múltiples respuestas por el método de la función de conveniencia para un diseño de mezclas," Revista Investigación Operacional, vol. 23, no. 1, pp. 84-90, Ene. 2002.

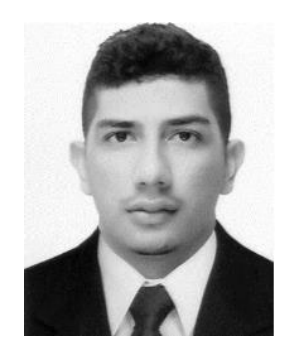

Kevin Farid Gil-Rodríguez nació en Barranquilla, Atlántico, Colombia en 1990. Recibió su título de Ingeniero Mecánico en la Universidad del Atlántico (Puerto Colombia, Colombia) en 2019. Sus áreas de interés investigativo incluyen el diseño de experimentos, diseño de elementos de máquinas y procesos de manufactura de productos cerámicos. Actualmente se desempeña como ingeniero de producción en la empresa Gres Caribe S.A. en Barranquilla (Colombia).

ORCID: https://orcid.org/0000-0002-6433-8567 


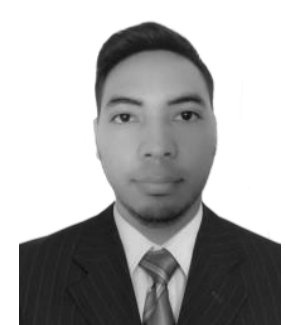

Jorddy Aldair Mendoza-Ruiz nació en Barranquilla, Atlántico, Colombia en 1994. Recibió su título de Ingeniero Mecánico en la Universidad del Atlántico (Puerto Colombia, Colombia) en 2019. Las áreas de interés investigativo incluyen el diseño de experimentos, diseño de elementos de máquinas y RCM. Actualmente se desempeña como coordinador del proyecto "Diagnóstico y obras de rehabilitación de la infraestructura del distrito de riego y drenaje de Repelón (Departamento del Atlántico)", en el área electromecánica con el Consorcio Repelón 2018.

ORCID: https://orcid.org/0000-0002-2854-569X

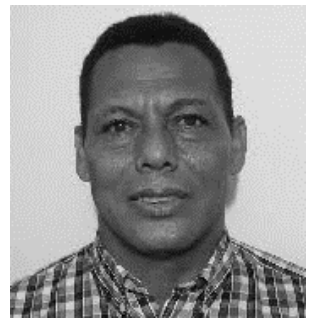

Antonio Saltarín Jiménez es Ingeniero Mecánico de la Universidad del Norte (Puerto Colombia, Colombia). Recibió título de Especialista en Gestión Eficiente de Energía por la Universidad del Atlántico (Puerto Colombia, Colombia) en el año 2002. Sus áreas de interés profesional e investigativo se centran principalmente en mantenimiento industrial, diseño de maquinaria y mejora energética de procesos. Actualmente se desempeña como consultor industrial y automotriz, así como docente de cátedra en la Universidad del Atlántico.

ORCID: https://orcid.org/0000-0003-1056-1699

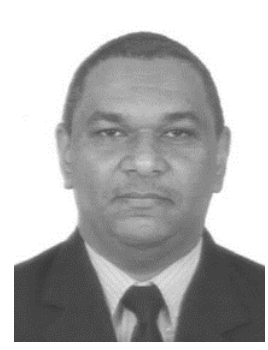

Milton Coba Salcedo nació en Barranquilla (Colombia). El recibió su título de Ingeniero Mecánico de la Universidad del Atlántico (Puerto Colombia, Colombia) en el año 2001, y título de Doctor en Ingeniería Mecánica de la Universidad Politécnica de Cataluña (España) en el año 2012. Actualmente es Profesor Asociado del Programa de Ingeniería Mecánica en la Universidad del Atlántico e integrante del Grupo IMTEF. Sus intereses investigativos incluyen principalmente procesos de manufactura y diseño de maquinaria.

ORCID: https://orcid.org/0000-0002-2436-5536

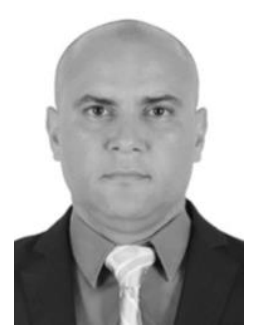

Javier Roldán Mckinley es Ingeniero Mecánico de la Universidad del Atlántico (Puerto Colombia, Colombia) en el año 2001. Obtuvo grado de Doctor en Ingeniería Mecánica en la Universidad de la Florida (Gainesville, FL-USA) en el año 2007. Actualmente trabaja como Profesor Asociado del Programa de Ingeniería Mecánica de la Universidad del Atlántico (Puerto Colombia, Colombia). Es integrante activo del grupo de investigación DIMER. Sus intereses investigativos incluyen principalmente la instrumentación y control de procesos $\mathrm{y}$ sistemas, diseño de mecanismos y máquinas, y educación de la ingeniería.

ORCID: https://orcid.org/0000-0003-1444-6460 